MATHEMATICS OF COMPUTATION

Volume 70, Number 235, Pages 911-934

S $0025-5718(00) 01250-3$

Article electronically published on March 24, 2000

\title{
ERROR ESTIMATES FOR THE THREE-FIELD FORMULATION WITH BUBBLE STABILIZATION
}

\author{
FRANCO BREZZI AND DONATELLA MARINI
}

\begin{abstract}
In this paper we prove convergence and error estimates for the socalled 3-field formulation using piecewise linear finite elements stabilized with boundary bubbles. Optimal error bounds are proved in $L^{2}$ and in the broken $H^{1}$ norm for the internal variable $u$, and in suitable weighted $L^{2}$ norms for the other variables $\lambda$ and $\psi$.
\end{abstract}

\section{INTRODUCTION}

The aim of this paper is to consider the effects of bubble stabilizations of the so-called three-field formulation for domain decomposition methods. Let us briefly recall it. Assume that we have to solve a linear elliptic problem whose variational formulation is

$$
\text { find } w \in V \text { such that } \quad a(w, v)=(f, v) \quad \forall v \in V,
$$

on a domain $\Omega$. We assume that the problem is associated to a second-order differential operator, so that the space $V$ will be a subspace of $H^{1}(\Omega)$. By splitting $\Omega$ into subdomains $\Omega^{s}$ one introduces suitable subspaces $V^{s}$ (to be made precise in the sequel) of $H^{1}\left(\Omega^{s}\right)$, and defines $M^{s}=H^{-1 / 2}\left(\partial \Omega^{s}\right), \Sigma=\bigcup_{s} \partial \Omega^{s}$, and $\Phi=\{$ traces on $\Sigma$ of the functions of $V\}$. Setting $V^{*}:=\prod_{s} V^{s}$ and $M^{*}:=\prod_{s} M^{s}$, the three-field formulation of (1.1) then reads

$$
\left\{\begin{array}{lll}
\text { find } u \in V^{*}, \lambda \in M^{*}, \text { and } \psi \in \Phi \text { such that } & \\
\text { i) } a_{s}\left(u^{s}, v\right)-\left\langle\lambda^{s}, v\right\rangle_{s}=(f, v)_{s} & \forall v \in V^{s}, & \forall s, \\
\text { ii) }\left\langle\mu^{s}, u_{s}\right\rangle_{s}=\left\langle\mu^{s}, \psi\right\rangle_{s} & \forall \mu^{s} \in M^{s}, & \forall s, \\
\text { iii) } \sum_{s}\left\langle\lambda^{s}, \varphi\right\rangle_{s}=0 & \forall \varphi \in \Phi &
\end{array}\right.
$$

(the nearly obvious meaning of $a_{s},\langle., .\rangle_{s}$ and $(., .)_{s}$ will be made precise in the next section).

This formulation was originally introduced in [8, [9], where it was proved that, under reasonable assumptions, problem (1.2) has a unique solution related to the solution of (1.1) by

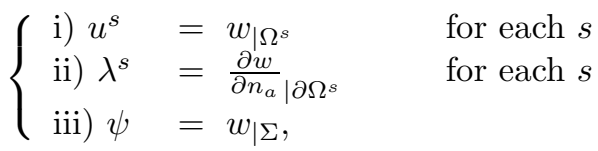

with $\left.\frac{\partial}{\partial n_{a}}\right|_{\partial \Omega^{s}}=$ outward conormal derivative to $\Omega^{s}$. In order to approximate (1.2) one has to choose, for every $s$, finite dimensional subspaces $V_{h}^{s}, M_{h}^{s}$ and $\Phi_{h}$ of

Received by the editor February 2, 1999 and, in revised form, August 5, 1999.

2000 Mathematics Subject Classification. Primary 65N55, 65N30, 65N12, 65N15, 35J25. 
$V^{s}, M^{s}$ and $\Phi$ respectively, to construct $V_{h}^{*}:=\prod_{s} V_{h}^{s}$ and $M_{h}^{*}:=\prod_{s} M_{h}^{s}$, and to consider the following discretized problem:

$$
\left\{\begin{array}{lll}
\text { find } u_{h} \in V_{h}^{*}, \lambda_{h} \in M_{h}^{*}, \text { and } \psi_{h} \in \Phi_{h} \text { such that } & \\
\text { i) } a_{s}\left(u_{h}^{s}, v\right)-\left\langle\lambda_{h}^{s}, v\right\rangle_{s}=(f, v)_{s} & \forall v \in V_{h}^{s}, & \forall s, \\
\text { ii) }\left\langle\mu^{s}, u_{h}^{s}\right\rangle_{s}=\left\langle\mu^{s}, \psi_{h}\right\rangle_{s} & \forall \mu^{s} \in M_{h}^{s}, & \forall s, \\
\text { iii) } \sum_{s}\left(\lambda_{h}^{s}, \varphi\right\rangle_{s}=0 & \forall \varphi \in \Phi_{h} . &
\end{array}\right.
$$

In [8], 9] we proved that a finite element discretization of (1.2) on compatible grids (with a suitable interpretation of $M_{h}^{s}$ ) gives back the global finite element solution of (1.1) with formulae identical to (1.3). To deal with more general approximations, including the use of (possibly) different discretizations for the three variables in the same subdomain, or from one subdomain to another, a wide class of stabilization procedures was introduced and discussed in [2]. However, it is interesting to see if, for particular cases, a simpler and cheaper stabilization could be used, instead of the more general (but heavier and costlier) stabilization of [2]. A typical example of interest is clearly the case where the variables $u$ and $\psi$ are approximated by continuous piecewise linear finite elements, although on different, incompatible grids, and the variable $\lambda$ by piecewise constants. This was done in [7, where a stabilization procedure was proposed, based on the idea of adding suitable boundary bubbles to the space $V_{h}^{s}$. The underlying concept is reasonably simple: it is clear that in (1.4) the only control on $\psi_{h}$ can be obtained from (1.4ii). Therefore, to gain control on $\psi_{h}$ one can increase the space $M_{h}^{s}$. As each space $M_{h}^{s}$ is made of piecewise constants, this can be done simply by refining the mesh. It has to be pointed out that, once the first refinement has been made, the grid for $M_{h}^{s}$ could undergo a further refinement (if convenient for some technical reason) without jeopardizing the control on $\psi_{h}$. In its turn, the control on $\lambda_{h}$ can only be obtained from equation (1.4 i), since (1.4 iii) is clearly too weak (any monster taking the same value with opposite signs at the interfaces will satisfy (1.4 iii)). Hence, to control $\lambda_{h}^{s}$ we have to enrich the finite element spaces $V_{h}^{s}$, and the cheapest way is to add boundary bubbles. In [7] we showed, on a single domain problem, that an almost arbitrary mismatch between the grid for $M_{h}^{s}$ and that for $V_{h}^{s}$ can be stabilized (to gain control on $\lambda_{h}^{s}$ ) by suitable quadratic boundary bubbles. We also showed that the bubbles, together with the unknown $\lambda_{h}$, could be easily eliminated by static condensation, thus remaining with a symmetric (if problem (1.1) is symmetric) positive definite problem in the unknown $u_{h}$ only. In particular, in [7] we assumed, as a starting point, that two original, unrelated grids were given, one for $\lambda_{h}$ and one for $u_{h}$. For technical reasons (essentially, in order to be able to perform the static condensation on $\lambda_{h}^{s}$ afterwards) our first step was to refine the grid for $M_{h}^{s}$, by adding all the boundary nodes of the grid for $V_{h}^{s}$. However we had to assume, to avoid technical difficulties, that the points of the two original grids were not too close (otherwise the refined grid for $M_{h}^{s}$ might contain very small intervals) and that the two given grids were "comparable" in the following sense:

$$
h_{\lambda}^{s} \geq \gamma h_{u}^{s}
$$

(with $\gamma$ a fixed constant), with obvious meaning for the symbols. These assumptions were removed or relaxed, always for the single domain case, in [10], where an optimal estimate for $u-u_{h}$ was proved, allowing the presence of "very close nodes" and 
changing (1.5) into the much weaker

$$
h_{\lambda}^{s} \geq \gamma\left(h_{u}^{s}\right)^{3}
$$

(see [10] for further details.)

Here we tackle the whole problem (1.4), proving optimal error estimates for the three variables $u, \lambda, \psi$. We assume that two independent grids are given, this time for $V_{h}^{s}$ and $\Phi_{h}$. It seems particularly reasonable to assume the grid for $\Phi_{h}$ as given, since in many cases this will be the space where multidomain preconditioners (for the Schur complement) will be applied: to have a convenient grid might then allow the use of more powerful preconditioners (see for instance [13], [15], 3], [5], 20], 14], [18, [19.)

Similarly, the given grids for $V_{h}^{s}$ might have been chosen, in each subdomain, to match specific difficulties arising in that subdomain, and related to the coefficients of the operator and/or to the right-hand side. On the other hand, the choice of the grid for $M_{h}^{s}$ seems to be less crucial, at least in some applications, and could be made in order to allow an easier stabilization. As in the single domain problem, we work with piecewise linear finite elements for $\psi_{h}$ and $u_{h}$. The bubbles used for stabilization, as well as the unknowns $\lambda_{h}^{s}$ (piecewise constants), can be eliminated by static condensation. For simplicity we restrict ourselves to the case were the given grids for $V_{h}^{s}$ and $\Phi_{h}$ are "comparable", in the above sense. It would surely be interesting to have results under weaker assumptions, at least of the type (1.6). On the other hand, we allow the case of "very close nodes" that can be generated by merging the nodes of the two given independent grids. For a multifield formulation similar to the one discussed here (but using four fields) and related preconditioners, we refer to [16].

We now give an outline of the paper. In the next section we make precise our assumptions on the domain, the operator, the given decompositions and the formulation of the stabilized discrete problem. We shall also recall a few trace theorems that will be used in the subsequent sections. In Section 3 we prove the error estimates: we start with $u-u_{h}$, proving optimal estimates first in the broken $H^{1}$-norm and then in the $L^{2}$-norm. Optimal error estimates are then proven for $\psi-\psi_{h}$ and for $\lambda-\lambda_{h}$, in suitable weighted $L^{2}$-norms. Under our assumptions, these estimates imply optimal error bounds in $L^{2}(\Sigma)$ for $\psi-\psi_{h}$. The same is true for $\lambda-\lambda_{h}$, but only outside the possible "very small intervals". Finally, in Section 4 we cover some numerical aspects, such as the actual implementation of the procedure and its expected numerical performance.

\section{Statement of the PROBlem AND DiscREtizAtion}

2.1. Assumptions on the operator, the domain, and the subdomains. To fix ideas, let us consider the following differential problem

$$
\begin{cases}A u=f & \text { in } \Omega \subset R^{2}, \\ u=0 & \text { on } \partial \Omega,\end{cases}
$$

where $\Omega$ is a convex polygon, and

$$
A u \equiv-\sum_{i, j=1}^{2} \frac{\partial}{\partial x_{j}}\left(a_{i j}(x) \frac{\partial u}{\partial x_{i}}\right)+a_{0}(x) u .
$$

We assume that $0 \leq a_{0}(x) \leq K$ for almost every $x$ in $\Omega$, and that the $2 \times 2$ matrix $\left(a_{i j}(x)\right)$ is symmetric and positive definite, with smallest eigenvalue $\geq \alpha>0$ and 
largest eigenvalue $\leq \beta$, independent of $x$. To the operator $A$ we assign the bilinear form

$$
a(w, v)=\int_{\Omega}\left(\sum_{i, j=1}^{2} a_{i j}(x) \frac{\partial w}{\partial x_{i}} \frac{\partial v}{\partial x_{j}}+a_{0}(x) w v\right) d x, \quad w, v \in H^{1}(\Omega) .
$$

Let $V=H_{0}^{1}(\Omega)$, with norm $\|\cdot\|_{V}=|\cdot|_{1, \Omega}$. With our assumptions on the coefficients the bilinear form is continuous and $V$-elliptic, i.e.,

$$
\begin{array}{rll}
\exists \alpha>0: & \alpha|v|_{1, \Omega}^{2} \leq a(v, v) & \forall v \in V, \\
\exists M>0: & a(w, v) \leq M|| w\left\|_{1, \Omega}\right\| v \|_{1, \Omega} & \forall w, v \in V .
\end{array}
$$

Then for smooth data, say, $f \in L^{2}(\Omega)$ and $a_{i j} \in C^{1}(\bar{\Omega})$, problem (1.1) has a unique solution $w \in H_{0}^{1}(\Omega) \cap H^{2}(\Omega)$.

Now let $\Omega$ be partitioned into a finite number $N$ of polygonal subdomains $\Omega^{s}$ $(s=1, \ldots, N)$, with boundary $\Gamma^{s}=\partial \Omega^{s}$ and diameter $H_{s}$. We make precise the notation of (1.2) by defining, for $s=1, \ldots, N$,

$$
\begin{aligned}
& V^{s}=\left\{v \in H^{1}\left(\Omega^{s}\right), v=0 \text { on } \Gamma^{s} \cap \partial \Omega\right\}, \\
& \langle\cdot, \cdot\rangle_{s}=\text { duality pairing between } H^{1 / 2}\left(\Gamma^{s}\right) \text { and } H^{-1 / 2}\left(\Gamma^{s}\right), \\
& (\cdot, \cdot)_{s}=\text { scalar product in } L^{2}\left(\Omega^{s}\right), \\
& a_{s}(w, v)=\sum_{i, j=1}^{2}\left(a_{i j}(x) \frac{\partial w}{\partial x_{i}}, \frac{\partial v}{\partial x_{j}}\right)_{s}+\left(a_{0}(x) w, v\right)_{s}, \quad w, v \in V .
\end{aligned}
$$

In agreement with the definition of $V$ and $\Phi=$ traces on $\Sigma$ of the functions of $V$ \}, we will have

$$
\Phi=\left\{\varphi \in H^{1 / 2}(\Sigma): \varphi=0 \text { on } \partial \Omega\right\} .
$$

With this notation problem (1.1) is equivalent to problem (1.2), and the relationship between the solution $w$ of (1.1) and the solution $(u, \lambda, \psi)$ of (1.2) is precisely given in (1.3). We explicitly note that (2.5) will also hold in each $\Omega^{s}$, if we replace $\Omega, V$ and $a$ with $\Omega^{s}, V^{s}$ and $a_{s}$ respectively.

Finally, we recall some known results related to trace theorems, whose proof can be checked by using trace theorems on the reference element. We have that for every $\theta_{0} \leq \pi / 3$ there exists a constant $C=C\left(\theta_{0}\right)$ such that, for every triangle $T$ with minimum angle bounded from below by $\theta_{0}$,

$$
\begin{aligned}
|v|_{1 / 2, \partial T} & \leq C|v|_{1, T} & & \forall v \in H^{1}(T), \\
\left|\frac{\partial v}{\partial n_{a}}\right|_{1 / 2, \partial T} & \leq C|v|_{2, T} & & \forall v \in H^{2}(T), \\
|v|_{3 / 2, \partial T} & \leq C|v|_{2, T} & & \forall v \in H^{2}(T),
\end{aligned}
$$

where, as we shall do throughout the whole paper, the usual notation for Sobolev norms and seminorms (see e.g. [12]) is used. On the other hand, we can easily deduce that, if $e$ is an edge of $T$, and $h_{T}$ its diameter,

$$
\|v\|_{0, e}^{2} \leq C h_{T}^{-1}\|v\|_{0, T}\left(\|v\|_{0, T}+h_{T}|v|_{1, T}\right) \quad \forall v \in H^{1}(T),
$$

always with a constant $C$ depending only on $\theta_{0}$. The basic instrument for proving (2.11) is the fundamental theorem of calculus applied to $v_{n}^{2}$, where $v_{n}$ is a sequence of smooth functions converging to $v$ in $H^{1}(T)$. 
We end this section by recalling a well known property of Sobolev fractional norms and seminorms. We report it for seminorms of fractional order $\theta$ in dimension one, but the result is more general. If an interval $I$ is the disjoint union of subintervals $I_{k}$, then

$$
\sum_{k}|v|_{\theta, I_{k}}^{2} \leq|v|_{\theta, I}^{2} \quad \forall v \in H^{\theta}(I)
$$

2.2. Assumptions on $\mathcal{T}_{\psi}, \mathcal{T}_{u}^{s}$ and generation of the $\mathcal{T}_{\lambda}^{s}$ decompositions. In order to discretize problem (1.2), let $\mathcal{T}_{\psi}$ be a decomposition of $\Sigma$ into intervals $I$, and for each $s=1, \ldots, N$ let $\mathcal{T}_{u}^{s}$ be a decomposition of $\Omega^{s}$ into triangles $T$, and $\mathcal{T}_{\lambda}^{s}$ a decomposition of $\Gamma^{s}$ into intervals $I$. We shall denote by $h_{u}^{s}, h_{\lambda}^{s}, h_{\psi}$ the mesh size of $\mathcal{T}_{u}^{s}, \mathcal{T}_{\lambda}^{s}, \mathcal{T}_{\psi}$ respectively. Next, we define the finite element spaces

$$
\begin{array}{rlrl}
V_{h}^{s} & =\left\{v \in V^{s}: v_{\mid T} \in P_{1}(T) \forall T \in \mathcal{T}_{u}^{s}\right\} & & \forall s, \\
M_{h}^{s} & =\left\{\mu^{s} \in L^{2}\left(\Gamma^{s}\right): \mu_{\mid I}^{s} \in P_{0}(I) \forall I \in \mathcal{T}_{\lambda}^{s}\right\} & \forall s, \\
\Phi_{h} & =\left\{\varphi \in \Phi: \varphi_{\mid I} \in P_{1}(I) \forall I \in \mathcal{T}_{\psi}\right\}, &
\end{array}
$$

and we set, as before, $V_{h}^{*}:=\prod_{s} V_{h}^{s}$ and $M_{h}^{*}:=\prod_{s} M_{h}^{s}$. Then we look for an approximate solution $\left(u_{h}, \lambda_{h}, \psi_{h}\right)$ of (1.2), with $u_{h} \in V_{h}^{*}, \lambda_{h} \in M_{h}^{*}$, and $\psi_{h} \in$ $\Phi_{h}$. If the three decompositions are totally arbitrary, the discrete problem can be unstable, and even singular. Here we assume that the decompositions $\mathcal{T}_{u}^{s}$ and $\mathcal{T}_{\psi}$ are given and 'untouchable'. To avoid unnecessary complications in the bubble stabilization described below, we just require that, for each $s$, the decomposition $\mathcal{T}_{u}^{s}$ does not include triangles having more than one edge on the boundary of $\Omega^{s}$. As we have seen in the Introduction, we can however choose the grid $\mathcal{T}_{\lambda}^{s}$ in such a way that the problem becomes stabilizable by the simple addition of boundary bubbles to the spaces $V_{h}^{s}$. To do that, however, we shall need, as in [7], some minor assumptions on the two decompositions $\mathcal{T}_{u}^{s}$ and $\mathcal{T}_{\psi}$. Next, we will generate the $\mathcal{T}_{\lambda}^{s}$ decomposition and introduce the stabilization through boundary bubbles. We assume that the decomposition $\mathcal{T}_{\psi}$ is quasi-uniform [12] and that, for all $s$, the restriction to the boundary $\Gamma^{s}$ of $\mathcal{T}_{u}^{s}$ is also quasi-uniform. We assume moreover that the decompositions are comparable. In particular we introduce $h_{\max }$ as the biggest among the lengths of the intervals of $\mathcal{T}_{\psi}$ and the lengths of the boundary edges of the decompositions $\mathcal{T}_{u}^{s}$, for all $s$. Similarly, we define $h_{\min }$ as the minimum over the same set. We assume that:

$$
\exists \gamma_{1}>0 \text { such that } h_{\max } \leq \gamma_{1} h_{\min } .
$$

In the sequel, to avoid heaviness in the notation we shall write $h$ in place of $h_{\min }$ and $\bar{h}$ for the maximum diameter over $\mathcal{T}_{\psi}$ and over all $\mathcal{T}_{u}^{s}$ 's (including internal triangles). We apologize for the rather unusual notation but, as we shall see, $h$ will be used much more often than $\bar{h}$.

Remark 1 . The assumptions on the $\mathcal{T}_{u}$ grid are rather heavy, and will forbid the application of the present theory to several interesting cases. We remark nevertheless that, in some cases, grid refinements and self-adaptive procedures might occur only in the interior of the subdomains, thus escaping our present limitations. On the other hand, as we shall see in the last section, we believe that the above assumptions are just technical ones, and that the results (and the applicability of the whole method) are valid in more general circumstances. 
We now generate the grid for the $\lambda_{h}$ 's as

$$
\mathcal{T}_{\lambda}^{s}=\operatorname{merge}\left\{\left(\mathcal{T}_{u}^{s}\right)_{\mid \Gamma^{s}},\left(\mathcal{T}_{\psi}\right)_{\mid \Gamma^{s}}\right\} \quad \forall s .
$$

More precisely, for all $s$, we take as nodes for $\mathcal{T}_{\lambda}^{s}$ the union of the nodes of $\mathcal{T}_{\psi}$ and the nodes of $\mathcal{T}_{u}^{s}$ belonging to $\Gamma^{s}$. In doing this it may occur that $\mathcal{T}_{\lambda}^{s}$ has very small intervals, whenever two nodes of $\left(\mathcal{T}_{u}^{s}\right)_{\mid \Gamma^{s}}$ and $\left(\mathcal{T}_{\psi}\right)_{\mid \Gamma^{s}}$ get too close. Such "small intervals" will be allowed in our discussion (neglecting possible programming problems), but will need a special treatment from the theoretical point of view. For this we introduce a constant $\beta$ such that $0<\beta<1$, and we say, following essentially the notation of [10], that the intervals whose length is smaller than $\beta h$ are "irregular". More precisely, for $I_{\lambda}^{s} \in \mathcal{T}_{\lambda}^{s}$, we say that

$$
I_{\lambda}^{s} \text { is "irregular" if }\left|I_{\lambda}^{s}\right| \leq \beta h .
$$

Otherwise $I_{\lambda}^{s}$ will be called "regular". Therefore, we have

$$
\exists \gamma_{2}>0 \text { such that, } \forall s \forall I_{\lambda}^{s} \in \mathcal{T}_{\lambda}^{s}, \quad \text { if } I_{\lambda}^{s} \text { is "regular", then }\left|I_{\lambda}^{s}\right| \geq \gamma_{2} h .
$$

Note that one endpoint of an irregular interval must be a node of $\mathcal{T}_{u}^{s}$, and the other one a node of $\mathcal{T}_{\psi}$.

2.3. Introduction of the bubbles. For each $s$, we add to the discretization of $u^{s}$ as many bubbles as the intervals of $\mathcal{T}_{\lambda}^{s}$. More precisely, let $T$ be a triangle having an edge $T^{\prime}$ on $\Gamma^{s}$, so that $T^{\prime}=\bigcup_{k} I_{k}, I_{k} \in \mathcal{T}_{\lambda}^{s}$. Accordingly, $T=\bigcup_{k} T_{k}$ (see Figure 1). A boundary bubble $b_{k}$ is a function $b_{k} \in H^{1}\left(\Omega^{s}\right)$ such that $\operatorname{supp}\left(b_{k}\right) \subset T_{k}$ and $\int_{I_{k}} b_{k} \neq 0$. (See [7] for more details.) The optimal shape of these bubbles is still under investigation. As a simple example, one can think of a quadratic function vanishing on the two internal edges of $T_{k}$ (see Figure 1). As the choice has to be made up to a scaling factor, we can then assume that $b_{k}$ has value $1 / 4$ at the midpoint of $I_{k}$. With this choice one can easily compute that

$$
\int_{T_{k}} b_{k} d x=\left|T_{k}\right| / 12, \quad \int_{I_{k}} b_{k} d s=\left|I_{k}\right| / 6, \quad \int_{T_{k}}\left|\underline{\nabla} b_{k}\right|^{2} d x=\frac{\sum_{i=1}^{3}\left|e_{k, i}\right|^{2}}{48\left|T_{k}\right|},
$$

where the $e_{k, i}$ are the edges of $T_{k}$. Consequently, we can deduce that

$$
\left|b_{k}\right|_{1, T_{k}}^{2} \equiv \int_{T_{k}}\left|\underline{\nabla} b_{k}\right|^{2} d x \leq \gamma h_{T} /\left|I_{k}\right|,
$$

with $h_{T}=$ diameter of $T,\left|I_{k}\right|=$ length of $I_{k}$, and $\gamma$ a positive constant independent of $h_{T}$ and $\left|I_{k}\right|$.
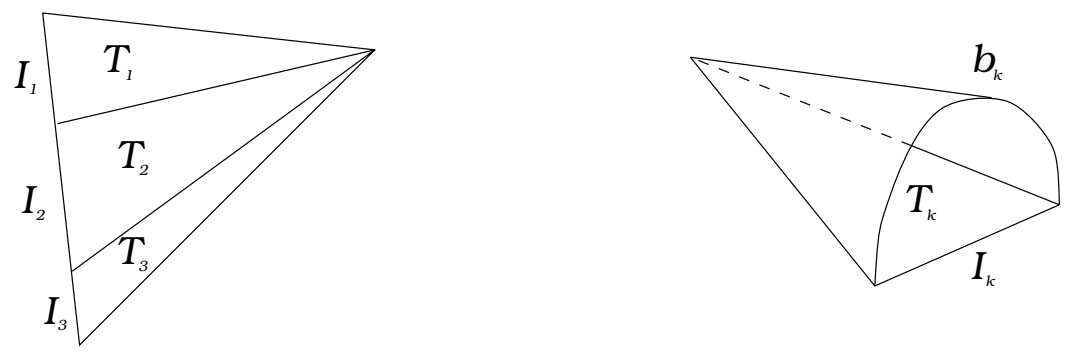

FIGURE 1. 
2.4. The stabilized problem. From now on we shall always assume that the decompositions $\mathcal{T}_{\psi}$ and $\mathcal{T}_{u}^{s}$ are given and satisfy (2.16), that the decompositions $\mathcal{T}_{\lambda}^{s}$ are generated according to (2.17), and that the spaces $V_{h}^{s}, M_{h}^{s}$ and $\Phi_{h}$ are defined as in (2.13), (2.14) and (2.15), respectively. Moreover, for every $s$, let $B_{h}^{s}$ be the space of bubbles introduced above. We shall replace the space $V_{h}^{s}$ by the augmented space

$$
\widetilde{V}_{h}^{s}=V_{h}^{s} \oplus B_{h}^{s} \quad \forall s
$$

and then set $\widetilde{V}_{h}^{*}=\prod_{s} \widetilde{V}_{h}^{s}, M_{h}^{*}=\prod_{s} M_{h}^{s}$. The stabilized discrete problem is now

$$
\begin{cases}\text { find } u_{h} \in \widetilde{V}_{h}^{*}, \lambda_{h} \in M_{h}^{*} \text { and } \psi_{h} \in \Phi_{h} \text { such that } \\ \text { i) } a_{s}\left(u_{h}^{s}, v\right)-\left\langle\lambda_{h}^{s}, v\right\rangle_{s}=(f, v)_{s}, & \forall v \in \widetilde{V}_{h}^{s}, \forall s \\ \text { ii) }\left\langle\mu^{s}, u_{h}^{s}\right\rangle_{s}=\left\langle\mu^{s}, \psi_{h}\right\rangle_{s}, & \forall \mu^{s} \in M_{h}^{s}, \forall s \\ \text { iii) } \sum_{s}\left\langle\lambda_{h}^{s}, \varphi\right\rangle_{s}=0 & \forall \varphi \in \Phi_{h} .\end{cases}
$$

Remark 2. As pointed out in [7] and further developed in [10] for the single-domain case, the bubbles and the multipliers $\lambda_{h}^{s}$ can be eliminated by static condensation, leaving, as unknowns, only $\psi_{h}$ and the piecewise linear part of $u_{h}$. This will be further discussed in the last section.

\section{ERror estimates}

Comparing (1.2) and (2.23) we easily obtain the error equations

$$
\begin{cases}\text { i) } a_{s}\left(u^{s}-u_{h}^{s}, v\right)-\left\langle\lambda^{s}-\lambda_{h}^{s}, v\right\rangle_{s}=0, & \forall v \in \widetilde{V}_{h}^{s} \forall s, \\ \text { ii) }\left\langle\mu^{s}, u^{s}-u_{h}^{s}\right\rangle_{s}=\left\langle\mu^{s}, \psi-\psi_{h}\right\rangle_{s}, & \forall \mu^{s} \in M_{h}^{s} \forall s, \\ \text { iii) } \sum_{s}\left\langle\lambda^{s}-\lambda_{h}^{s}, \varphi\right\rangle_{s}=0, & \forall \varphi \in \Phi_{h} .\end{cases}
$$

Let $u_{I}^{s} \in \widetilde{V}_{h}^{s}, \lambda_{I}^{s} \in M_{h}^{s}, \psi_{I} \in \Phi_{h}$ be some interpolants of $u^{s} \in V^{s}, \lambda^{s} \in M^{s}, \psi \in \Phi$ respectively, to be specified later. Then, by adding and subtracting these interpolants in (3.1) we obtain

$$
\begin{aligned}
\alpha \sum_{s}\left|u^{s}-u_{h}^{s}\right|_{1, \Omega^{s}}^{2} & \\
\leq & \underbrace{\sum_{s} a_{s}\left(u^{s}-u_{h}^{s}, u^{s}-u_{I}^{s}\right)}_{I}+\sum_{s} a_{s}\left(u^{s}-u_{h}^{s}, u_{I}^{s}-u_{h}^{s}\right) \quad(\text { use }(\underline{3.1} i)) \\
= & +\sum_{s}\left\langle\lambda^{s}-\lambda_{h}^{s}, u_{I}^{s}-u_{h}^{s}\right\rangle_{s} .
\end{aligned}
$$

For the second term in (3.2), by adding and subtracting $u^{s}$ we have

$$
\begin{aligned}
\sum_{s}\left\langle\lambda^{s}-\lambda_{h}^{s}, u_{I}^{s}-u_{h}^{s}\right\rangle_{s} & \\
= & \underbrace{\sum_{s}\left\langle\lambda^{s}-\lambda_{h}^{s}, u_{I}^{s}-u^{s}\right\rangle_{s}}_{I I}+\sum_{s}\left\langle\lambda^{s}-\lambda_{h}^{s}, u^{s}-u_{h}^{s}\right\rangle_{s} \\
= & +\sum_{s}\left\langle\lambda^{s}-\lambda_{h}^{s}, u^{s}-u_{h}^{s}\right\rangle_{s} .
\end{aligned}
$$


By inserting $\lambda_{I}^{s}$ in the last term of (3.3) and using (3.1) ii), we now have

$$
\begin{aligned}
& \sum_{s}\left\langle\lambda^{s}-\lambda_{h}^{s}, u^{s}-u_{h}^{s}\right\rangle_{s} \\
&=\underbrace{\sum_{s}\left\langle\lambda^{s}-\lambda_{I}^{s}, u^{s}-u_{h}^{s}\right\rangle_{s}}_{I I I}+\sum_{s}\left\langle\lambda_{I}^{s}-\lambda_{h}^{s}, u^{s}-u_{h}^{s}\right\rangle_{s} \\
&=\sum_{s}\left\langle\lambda_{I}^{s}-\lambda_{h}^{s}, \psi-\psi_{h}\right\rangle_{s} .
\end{aligned}
$$

Finally, inserting $\lambda^{s}$ in the last term of (3.4) and using (3.1) iii), we have

$$
\begin{aligned}
\sum_{s}\left\langle\lambda_{I}^{s}-\lambda_{h}^{s}, \psi-\psi_{h}\right\rangle_{s} & \\
= & =\underbrace{\sum_{s}\left\langle\lambda_{I}^{s}-\lambda^{s}, \psi-\psi_{h}\right\rangle_{s}}_{I V}+\underbrace{\sum_{s}\left\langle\lambda^{s}-\lambda_{h}^{s}, \psi-\psi_{I}\right\rangle_{s}}_{V .}
\end{aligned}
$$

We will now define proper interpolants to estimate the different pieces of (3.2), (3.3), (3.4), and (3.5). From now on we will omit the superscript $s$ unless it is really necessary.

Let $\psi_{I} \in \Phi_{h}$ be the usual continuous piecewise linear interpolant of $\psi$, and, for each $s$, let $u_{I} \in V_{h}^{s}$ be the usual continuous piecewise linear interpolant of $u$. The standard interpolation estimates are [12]

$$
\begin{aligned}
& \| u-\left.u_{I}\right|_{r, T} \leq C h_{T}^{2-r}|u|_{2, T} \quad \forall T \in \mathcal{T}_{u}^{s} \quad(r=0,1), \\
& \| u-\left.u_{I}\right|_{r, e} \leq C|e|^{3 / 2-r}|u|_{3 / 2, e} \quad \forall e \text { edge of } T, \forall T \in \mathcal{T}_{u}^{s} \quad(r=0,1) \text {, } \\
& \| \psi-\psi_{I}||_{r, I} \leq C|I|^{3 / 2-r}|\psi|_{3 / 2, I} \quad \forall I \in \mathcal{T}_{\psi} \quad(r=0,1) .
\end{aligned}
$$

(Here and in the sequel $C$ will denote a constant independent of the mesh size, not necessarily the same in the various occurrences.) For the $\lambda$-variable two different 'interpolants' will be needed. For each $s$, let us denote by $\lambda_{I}$ the piecewise constant function on the $\mathcal{T}_{\psi \text {-grid }}$ defined by

$$
\int_{I_{\psi}}\left(\lambda-\lambda_{I}\right) d s=0 \quad \forall I_{\psi} \in \mathcal{T}_{\psi},
$$

and by $\widetilde{\lambda} \in M_{h}^{s}$ the piecewise constant function on the $\mathcal{T}_{\lambda}$-grid defined by

$$
\int_{I_{\lambda}^{s}}(\lambda-\widetilde{\lambda}) b d s=0 \quad \forall I_{\lambda}^{s} \in M_{h}^{s}, \forall b \in B_{h}^{s} .
$$

We recall that the following interpolation estimates hold (see [12]):

$$
\begin{array}{cc}
\left\|\lambda-\lambda_{I}\right\|_{-r, I_{\psi}} \leq C\left|I_{\psi}\right|^{1 / 2+r}|\lambda|_{1 / 2, I_{\psi}}, & 0 \leq r \leq 1 / 2, \\
\| \lambda-\left.\tilde{\lambda}\right|_{-r, I_{\lambda}^{s}} \leq C\left|I_{\lambda}^{s}\right|^{1 / 2+r}|\lambda|_{1 / 2, I_{\lambda}^{s}}, & 0 \leq r \leq 1 / 2 .
\end{array}
$$

We also recall that (1.3) implies that $\lambda$ takes opposite values on the two sides of each subdomain interface. In its turn $\lambda_{I}$, being defined on the $\mathcal{T}_{\psi}$-grid, inherits the same property. Hence,

$$
\sum_{s}\langle\lambda, \varphi\rangle_{s}=\sum_{s}\left\langle\lambda_{I}, \varphi\right\rangle_{s}=0 \quad \forall \varphi \in \Phi
$$

as $\varphi \in \Phi$ is single-valued on $\Sigma$. On the other hand, $\widetilde{\lambda}$ does not enjoy this property. 
Some comments are in order before proceeding to estimate the various pieces in (3.2) - (3.5). In doing this, two types of difficulties will arise. One is connected with the fact that, on the left-hand side of (3.2), we have the broken $H^{1}$-seminorm of the error while, in estimating some of the pieces, it would be easier to use the broken $H^{1}$-norm. Hence, we will need to bound the norm of the error with its seminorm. This is not a major problem, and is dealt with in Lemma 4. The second source of difficulties lies in the estimate of pieces $I I$ and $V$, whose treatment is actually very similar, where an estimate of $\lambda-\lambda_{h}$ in terms of $u-u_{h}$ is needed. This is done in Lemma 1.

Lemma 1. Let $T$ be a boundary triangle of $\mathcal{T}_{u}^{s}$, and let $I_{k}$ be a boundary edge as in Figure 1. Then we have

$$
\left\|\lambda_{h}-\tilde{\lambda}\right\|_{0, I_{k}} \leq C h_{T}^{1 / 2}\left|I_{k}\right|^{-1}|| u-u_{h} \|_{1, T_{k}}
$$

where $(u, \lambda, \psi)$ is the solution of (1.2) and $\left(u_{h}, \lambda_{h}, \psi_{h}\right)$ that of 2.23).

Proof. We have

$$
\begin{aligned}
& \left.\left|\lambda_{h}-\tilde{\lambda}\right|_{\mid I_{k}}=\left|\int_{I_{k}}\left(\lambda_{h}-\tilde{\lambda}\right) b_{k} d s\right| / \int_{I_{k}} b_{k} d s \quad \text { (use (13.10) }\right) \\
& \left.\left.=\left|\int_{I_{k}}\left(\lambda_{h}-\lambda\right) b_{k} d s\right| / \int_{I_{k}} b_{k} d s \quad \text { (use (3.1 } i\right)\right) \\
& =\left|a\left(u-u_{h}, b_{k}\right)\right| / \int_{I_{k}} b_{k} d s \quad \text { (use (2.5)) } \\
& \leq M\left\|u-u_{h}\right\|_{1, T_{k}}\left\|b_{k}\right\|_{1, T_{k}} / \int_{I_{k}} b_{k} d s \quad \text { (use (2.20)-(2.21) } \\
& \leq C h_{T}^{1 / 2}\left|I_{k}\right|^{-3 / 2}|| u-u_{h} \|_{1, T_{k}} .
\end{aligned}
$$

The result follows by observing that

$$
\| \lambda_{h}-\left.\widetilde{\lambda}\right|_{0, I_{k}}=\left|\lambda_{h}-\widetilde{\lambda}\right|_{\mid I_{k}}\left|I_{k}\right|^{1 / 2}
$$

The unsatisfactory part of 3.14 is clearly the presence of $\left|I_{k}\right|^{-1}$. To deal with it in the treatment of $I I$ and $V$ we shall need an estimate for $u-u_{I}$ and $\psi-\psi_{I}$, respectively, where $\left|I_{k}\right|$ appears as a factor. This is done in the following lemma.

Lemma 2. Let $e \subset \Gamma^{s}$ be either an edge of a triangle of $\mathcal{T}_{u}^{s}$ or an interval of $\mathcal{T}_{\psi}$, and let $I_{k}$ be an interval of $\mathcal{T}_{\lambda}^{s}$ included in $e$. Let $g$ be a function in $H^{3 / 2}(e)$, and let $g^{I}$ be its linear interpolant on $e$ (that is, $g^{I} \in P_{1}(e)$, and $g^{I}=g$ at the endpoints of e). Then

$$
\left\|g-g_{I}\right\|_{0, I_{k}} \leq C|e|^{1 / 2}\left|I_{k}\right||g|_{3 / 2, e}
$$

Proof. If $I_{k}$ is a regular interval, the result follows immediately from standard interpolation estimates 12 and (2.16), 2.19). Indeed, we have

$$
\| g-\left.g_{I}\right|_{0, I_{k}} \leq C|e|^{3 / 2}|g|_{3 / 2, e} \leq C|e|^{1 / 2}\left|I_{k}\right||g|_{3 / 2, e} .
$$

If instead $I_{k}$ is irregular, then one of its endpoints must coincide with an endpoint of $e$. Setting then $d=g-g_{I}$, and choosing a coordinate system on $e$ such that 
$I_{k}=\left(0,\left|I_{k}\right|\right)$ and $d(0)=0$, from the fundamental theorem of calculus and the Cauchy-Schwarz inequality we get

$$
d(s)=\int_{0}^{s} d^{\prime}(t) d t \leq s^{1 / 2}\left(\int_{0}^{s}\left(d^{\prime}(t)\right)^{2} d t\right)^{1 / 2} .
$$

From (3.19) we then have

$$
\begin{aligned}
\|d\|_{0, I_{k}}^{2} & \equiv \int_{0}^{\left|I_{k}\right|} d^{2}(s) d s \leq \int_{0}^{\left|I_{k}\right|} s\left(\int_{0}^{s}\left(d^{\prime}(t)\right)^{2} d t\right) d s \\
& \leq\left|I_{k}\right| \int_{0}^{\left|I_{k}\right|}\left(\int_{0}^{\left|I_{k}\right|}\left(d^{\prime}(t)\right)^{2} d t\right) d s=\left|I_{k}\right|^{2}|d|_{1, I_{k}}^{2},
\end{aligned}
$$

so that

$$
|| g-\left.g_{I}\right|_{0, I_{k}} \leq\left|I_{k}\right|\left|g-g_{I}\right|_{1, I_{k}} .
$$

Finally, the result follows from (3.21) and interpolation estimates [12]:

$$
\left|g-g_{I}\right|_{1, I_{k}} \leq\left|g-g_{I}\right|_{1, e} \leq C|e|^{1 / 2}|g|_{3 / 2, e} .
$$

Lemma 2 is the crucial step where we could not avoid the assumption (2.16), at least in the present line of proof. Indeed, for intervals $I_{k}$ which are away from the endpoints of $e$, we cannot estimate ||$g-\left.g_{I}\right|_{0, I_{k}}$ better than $|e|^{2}\left|I_{k}\right|^{1 / 2}$, even using a $W^{2, \infty}$ regularity for $g$ (which, in the applications, will be either $u$ or $\psi$.) This is not enough to compensate for the $\left|I_{k}\right|^{-1}$ term in (3.14), unless $|e|$ is bounded in terms of $\left|I_{k}\right|$. The use of a higher regularity for $g$ and/or a nonlinear bound for $|e|$ in terms of $\left|I_{k}\right|$ would greatly increase the ugliness of the paper without seriously improving the quality of the results. An ideal result would be to derive optimal error bounds without assuming any relationship between $\mathcal{T}_{u \mid \Sigma}$ and $\mathcal{T}_{\psi}$, but we have not succeeded in that so far. In view of this, from now on we shall use (2.16) whenever this can simplify the proofs, even when it is not strictly necessary.

The combined use of Lemma 1 and Lemma 2 allows us to prove the following Lemma 3 and Corollary 1, which provide the basic instruments for estimating the terms $I I$ and $V$, respectively.

Lemma 3. Let $T$ be a boundary triangle of $\mathcal{T}_{u}^{s}$ with a boundary edge $T^{\prime}$, and let $I_{k}$ be an interval of $\mathcal{T}_{\lambda}^{s}$ included in $T^{\prime}$. Then

$$
\left|\int_{I_{k}}\left(\lambda-\lambda_{h}\right)\left(u-u_{I}\right) d s\right| \leq C\left(h_{T}^{2}|\lambda|_{1 / 2, I_{k}}+h_{T}|| u-u_{h} \|_{1, T}\right)|u|_{2, T},
$$

where $(u, \lambda, \psi)$ is the solution of (1.2), $\left(u_{h}, \lambda_{h}, \psi_{h}\right)$ that of (2.23), and $u_{I}$ is the linear interpolant of $u$.

Proof. Using the Cauchy-Schwarz inequality, the triangle inequality, the interpolation estimate (3.12) and then (3.14) and (3.17) with $g=u$, we easily deduce 
that

$$
\begin{aligned}
& \left|\int_{I_{k}}\left(\lambda-\lambda_{h}\right)\left(u-u_{I}\right) d s\right| \leq\left\|\lambda-\left.\lambda_{h}\right|_{0, I_{k}}|| u-u_{I}\right\|_{0, I_{k}} \\
& \quad \leq\left(|| \lambda-\widetilde{\lambda}\left\|_{0, I_{k}}+\right\| \widetilde{\lambda}-\lambda_{h} \|_{0, I_{k}}\right) \| u-\left.u_{I}\right|_{0, I_{k}} \\
& \quad \leq\left(C\left|I_{k}\right|^{1 / 2}|\lambda|_{1 / 2, I_{k}}+\left\|\widetilde{\lambda}-\lambda_{h}\right\|_{0, I_{k}}\right)|| u-\left.u_{I}\right|_{0, I_{k}} \\
& \quad \leq C\left(\left|I_{k}\right|^{1 / 2}|\lambda|_{1 / 2, I_{k}}+h_{T}^{1 / 2}\left|I_{k}\right|^{-1}|| u-u_{h} \|_{1, T}\right) h_{T}^{1 / 2}\left|I_{k}\right||u|_{3 / 2, T^{\prime}}
\end{aligned}
$$

and the result follows immediately from (2.10) and $\left|I_{k}\right| \leq C h_{T}$.

With similar arguments we can prove the following result.

Corollary 1. Under the hypotheses of Lemma 3 we have

$$
\left|\int_{I_{k}}\left(\lambda-\lambda_{h}\right)\left(\psi-\psi_{I}\right) d s\right| \leq C\left(\left|I_{k}\right|^{3 / 2}|\lambda|_{1 / 2, I_{k}}+h_{T}^{1 / 2}|| u-u_{h} \|_{1, T}\right)\left|I_{\psi}\right|^{1 / 2}|\psi|_{3 / 2, I_{\psi}},
$$

where $(u, \lambda, \psi)$ is the solution of (1.2), $\left(u_{h}, \lambda_{h}, \psi_{h}\right)$ that of (2.23), $I_{\psi}$ is the interval $\in \mathcal{T}_{\psi}$ such that $I_{k} \subseteq I_{\psi}$, and $\psi_{I}$ is the linear interpolant of $\psi$.

Proof. The proof mimics exactly that of Lemma 3 with $u=\psi, u_{I}=\psi_{I}$.

We now have all the necessary instruments to estimate the pieces (3.2)-(3.5). To simplify the following notation, we set, for $s=1, \ldots, N$,

$$
\begin{aligned}
E_{h, s}^{2} & =\left\|u-u_{h}\right\|_{1, \Omega^{s}}^{2} ; & E_{I, s}^{2} & =\sum_{T \subset \Omega^{s}} h_{T}^{2}|u|_{2, T}^{2} ; \\
E_{h}^{2} & =\sum_{s} E_{h, s}^{2} ; & E_{I}^{2} & =\sum_{s} E_{I, s}^{2} .
\end{aligned}
$$

We can now estimate the different pieces in (3.2)- (3.5). We begin with (3.2):

$$
\begin{aligned}
& \left.I=\sum_{s} a_{s}\left(u-u_{h}, u-u_{I}\right) \quad \text { (use (2.5) }\right) \\
& \leq M \sum_{s}\left\|u-u_{h}\right\|_{1, \Omega^{s}}\left\|u-u_{I}\right\|_{1, \Omega^{s}} \quad \text { (C-S) } \\
& \text { (3.28) } \left.\leq M\left(\sum_{s}\left\|u-u_{h}\right\|_{1, \Omega^{s}}^{2}\right)^{1 / 2}\left(\sum_{s}\left\|u-u_{I}\right\|_{1, \Omega^{s}}^{2}\right)^{1 / 2} \quad \text { (use (3.26) } \text { and (3.27) }\right) \\
& =M E_{h} E_{I} \text {; }
\end{aligned}
$$

C-S indicates the use of the Cauchy-Schwarz inequality. We now estimate (3.3). We recall that (2.16) implies that for every triangle $T$ in $\mathcal{T}_{u}^{s}$ having an edge $T^{\prime}$ in 
$\Gamma^{s}$ there are only a finite number of subintervals $I_{k}$ of $\mathcal{T}_{\lambda}^{s}$ contained in $T^{\prime}$. Then

$$
\begin{aligned}
I I & =\sum_{s}\left\langle\lambda-\lambda_{h}, u_{I}-u\right\rangle_{s} \\
& \left.=\sum_{s} \sum_{T} \sum_{k} \int_{I_{k}}\left(\lambda-\lambda_{h}\right)\left(u_{I}-u\right) d \Gamma \quad \text { (use (13.23) and (2.12) }\right) \\
& \leq C \sum_{s} \sum_{T}\left(h_{T}^{2}|\lambda|_{1 / 2, T^{\prime}}+h_{T}|| u-u_{h} \|_{1, T}\right)|u|_{2, T} \quad \text { (use (1.3) and (2.9)) } \\
& \leq C \sum_{s} \sum_{T}\left(h_{T}^{2}|u|_{2, T}+h_{T}|| u-u_{h} \|_{1, T}\right)|u|_{2, T} \quad \text { (C-S) } \\
& \leq C\left(E_{I}^{2}+E_{h} E_{I}\right) .
\end{aligned}
$$

The estimate of (3.4) is also simple, provided that we introduce the following notation. For every $s$, and for every $I_{\psi}$ in $\Gamma^{s}$ we denote by $T_{\psi}$ a generic triangle (not necessarily in $\mathcal{T}_{u}^{s}$ but respecting the minimum angle condition) belonging to $\Omega^{s}$ and having $I_{\psi}$ as an edge. Then

$$
\begin{aligned}
I I I & =\sum_{s}\left\langle\lambda-\lambda_{I}, u-u_{h}\right\rangle_{s} \\
& =\sum_{s} \sum_{I_{\psi}} \int\left(\lambda-\lambda_{I}\right)\left(u-u_{h}\right) d \Gamma \quad \text { ([3.9) and duality) } \\
& \leq \sum_{s} \sum_{I_{\psi}}|| \lambda-\left.\lambda_{I}\right|_{-1 / 2, I_{\psi}}\left|u-u_{h}\right|_{1 / 2, I_{\psi}} \quad \text { (use (3.11) and (2.8)) } \\
& \leq C \sum_{s} \sum_{I_{\psi}}\left|I_{\psi}\right||\lambda|_{1 / 2, I_{\psi}}\left|u-u_{h}\right|_{1, T_{\psi}} \quad \text { (use (2.9) and C-S) } \\
& \leq C \sum_{s}\left(\sum_{T} h_{T}^{2}|u|_{2, T}\right)^{1 / 2}\left|u-u_{h}\right|_{1, \Omega^{s}} \\
& \leq C E_{I} E_{h} .
\end{aligned}
$$

The term $I V$ would be a potential source of major difficulties, as it requires in principle an estimate of $\psi-\psi_{h}$ in terms of $u-u_{h}$. Our choice of $\lambda_{I}$, however, makes it vanish thanks to (3.13):

$$
I V=\sum_{s}\left\langle\lambda_{I}^{s}-\lambda^{s}, \psi-\psi_{h}\right\rangle_{s}=0 .
$$

Before estimating $V$ we need further notation. For every triangle $T$ in $\mathcal{T}_{u}^{s}$, we define $I_{\psi}(T)$ as the union of the intervals $I_{\psi}$ in $\mathcal{T}_{\psi}$ which intersect $\partial T$ on a set of nonzero length. Note that there are only a finite number of them, depending only on the constant $\gamma_{1}$ in (2.16). We also denote by $T_{\psi}^{s}$ any triangle (with the minimum angle property) in $\Omega^{s}$ having $I_{\psi}(T)$ as an edge. Then the estimate of the last piece proceeds as follows. Using successively (3.25), (2.12) and (2.16), (2.9), and (2.10), 
and the C-S inequality we have

$$
\begin{aligned}
V & =\sum_{s}\left\langle\lambda-\lambda_{h}, \psi-\psi_{I}\right\rangle_{s} \\
& =\sum_{s} \sum_{T} \sum_{k} \int_{I_{k}}\left(\lambda-\lambda_{h}\right)\left(\psi-\psi_{I}\right) d \Gamma \\
& \leq C \sum_{s} \sum_{T} \sum_{k}\left(\left|I_{k}\right|^{3 / 2}|\lambda|_{1 / 2, I_{k}}+h_{T}^{1 / 2}|| u-\left.u_{h}\right|_{1, T}\right)\left|I_{\psi}\right|^{1 / 2}|\psi|_{3 / 2, I_{\psi}} \\
& \leq C \sum_{s} \sum_{T}\left(h_{T}^{2}|\lambda|_{1 / 2, T^{\prime}}+h_{T}|| u-u_{h}||_{1, T}\right)|\psi|_{3 / 2, I_{\psi}(T)} \\
& \leq C \sum_{s} \sum_{T}\left(h_{T}^{2}|u|_{2, T}+h_{T}|| u-u_{h} \|_{1, T}\right)|u|_{2, T_{\psi}} \\
& \leq C\left(E_{I}^{2}+E_{h} E_{I}\right) .
\end{aligned}
$$

We can now collect equations (3.2)-(3.5) and the estimates (3.28)-3.32), thus obtaining

$$
\alpha \sum_{s}\left|u_{s}-u_{h}^{s}\right|_{1, \Omega^{s}}^{2} \leq C\left(E_{I}^{2}+E_{I} E_{h}\right) .
$$

The final estimate would then be achieved if we could bound the term $E_{h}=$ $\sum_{s}\left\|u-u_{h}\right\|_{1, \Omega^{s}}^{2}$ appearing in the right-hand side of (3.33) in terms of the lefthand side. For this we need the following lemma.

Lemma 4. If $(u, \lambda, \psi)$ and $\left(u_{h}, \lambda_{h}, \psi_{h}\right)$ are the solutions of (1.2) and (2.23) respectively, then

$$
\sum_{s}\left\|u-u_{h}\right\|_{1, \Omega^{s}}^{2} \leq C \sum_{s}\left|u-u_{h}\right|_{1, \Omega^{s}}^{2} .
$$

Proof. For each $s$, let $q_{s}$ be defined as

$$
q_{s}=\left(\int_{\Omega^{s}}\left(u-u_{h}\right) d x\right) /\left|\Omega^{s}\right|
$$

and let $q$ be the piecewise constant function defined on $\Omega$ by $q_{\mid \Omega^{s}}=q_{s}$. Then set

$$
w=u-u_{h}-q .
$$

Since $w$ has zero mean value in each $\Omega^{s}$, we easily have

$$
\sum_{s}\|w\|_{1, \Omega^{s}}^{2} \leq C \sum_{s}|w|_{1, \Omega^{s}}^{2}=C \sum_{s}\left|u-u_{h}\right|_{1, \Omega^{s}}^{2} .
$$

Now let $\chi \in H_{0}^{1}(\Omega) \cap H^{2}(\Omega)$ be the solution of $-\Delta \chi=q$ in $\Omega$; set $\tau=\nabla \chi$, and for every $s=1, \ldots, N$, set $\mu_{s}^{*}=\frac{\partial \chi}{\partial n_{s} \mid \Gamma^{s}}=\tau \cdot n_{s \mid \Gamma^{s}}$. We now consider a new, artificial triangulation in each $\Omega^{s}$ that agrees with $\mathcal{T}_{\psi}$ on $\Gamma^{s}$ and has maximum diameter smaller than or equal to $\bar{h}$ (plus, clearly, the usual minimum angle condition). Note 
that, in this way, we obtain a compatible decomposition of the whole $\Omega$. Now let $\tau^{I}$ be the lowest order Raviart-Thomas interpolant of $\tau$ (see e.g. [6]). We have

$$
\left\|\tau^{I}\right\|_{H(d i v ; \Omega)} \leq C\|\tau\|_{1, \Omega} \leq C\|\chi\|_{2, \Omega} \leq C\|q\|_{0, \Omega} .
$$

We notice that, for each $s$, if $\mu_{I}^{s}$ is the interpolant of $\mu_{s}^{*}$ defined as in (3.9), then

$$
\mu_{I}^{s}=\tau^{I} \cdot n_{s \mid \Gamma^{s}} .
$$

By classical arguments we then have

$$
\begin{aligned}
\|q\|_{0, \Omega}^{2} & =-\int_{\Omega} \Delta \chi q d x=-\sum_{s} \int_{\Omega^{s}} \Delta \chi q_{s} d x \\
& =-\sum_{s} \int_{\Gamma^{s}} \mu_{s}^{*} q_{s} d \Gamma=-\sum_{s} \int_{\Gamma^{s}} \mu_{I}^{s} q_{s} d \Gamma \\
& =-\sum_{s} \int_{\Gamma^{s}} \mu_{I}^{s}\left(q_{s}+w\right) d \Gamma+\sum_{s} \int_{\Gamma^{s}} \mu_{I}^{s} w d \Gamma \\
& =-\sum_{s} \int_{\Gamma^{s}} \mu_{I}^{s}\left(u-u_{h}\right) d \Gamma+\sum_{s} \int_{\Gamma^{s}} \mu_{I}^{s} w d \Gamma \\
& =-\sum_{s} \int_{\Gamma^{s}} \mu_{I}^{s}\left(\psi-\psi_{h}\right) d \Gamma+\sum_{s} \int_{\Gamma^{s}} \mu_{I}^{s} w d \Gamma .
\end{aligned}
$$

The first term in the last line of (3.40) vanishes thanks to (3.13), and the second can be bounded as follows:

$$
\begin{aligned}
\sum_{s} \int_{\Gamma^{s}} \mu_{I}^{s} w d \Gamma & =\sum_{s} \int_{\Gamma^{s}} \tau^{I} \cdot n_{s} w d \Gamma=\sum_{s}\left(\int_{\Omega^{s}} \operatorname{div} \tau^{I} w d x+\int_{\Omega^{s}} \tau^{I} \cdot \nabla w d x\right) \\
& \leq\left\|\tau^{I}\right\|_{H(d i v ; \Omega)}\left(\sum_{s}\|w\|_{1, \Omega^{s}}^{2}\right)^{1 / 2} \leq C\|q\|_{0, \Omega}\left(\sum_{s}\|w\|_{1, \Omega^{s}}^{2}\right)^{1 / 2},
\end{aligned}
$$

where in the first step we used (3.39), and in the last step we used (3.38). Hence, from (3.40), (3.41) and (3.37),

$$
\|q\|_{0, \Omega} \leq C\left(\sum_{s}\|w\|_{1, \Omega^{s}}^{2}\right)^{1 / 2} \leq C\left(\sum_{s}\left|u-u_{h}\right|_{1, \Omega^{s}}^{2}\right)^{1 / 2} .
$$

Since

$$
\sum_{s}\left\|u-u_{h}\right\|_{1, \Omega^{s}}^{2}=\sum_{s}\|w+q\|_{1, \Omega^{s}}^{2}=\sum_{s}\left(\|w\|_{1, \Omega^{s}}^{2}+\|q\|_{0, \Omega^{s}}^{2}\right),
$$

using (3.42) in (3.43) we have the result.

From (3.33) and (3.34) we deduce the first convergence theorem.

Theorem 1. Let $(u, \lambda, \psi)$ and $\left(u_{h}, \lambda_{h}, \psi_{h}\right)$ be the solutions of (1.2) and (2.23) respectively. Then

$$
E_{h}=\left(\sum_{s}\left\|u-u_{h}\right\|_{1, \Omega^{s}}^{2}\right)^{1 / 2} \leq C E_{I} \leq C \bar{h}|u|_{2, \Omega} .
$$

We are now able to prove a convergence result in $L^{2}(\Omega)$ for the $u$ variable. 
Theorem 2. Let $(u, \lambda, \psi)$ and $\left(u_{h}, \lambda_{h}, \psi_{h}\right)$ be the solutions of (1.21) and (2.23), respectively. Then

$$
\| u-\left.u_{h}\right|_{0, \Omega} \leq C \bar{h} E_{I} \leq C \bar{h}^{2}|u|_{2, \Omega} .
$$

Proof. Let $w \in H_{0}^{1}(\Omega) \cap H^{2}(\Omega)$ be the solution of the adjoint problem of (2.1), $A^{*} w=u-u_{h}$ in $\Omega$. For every $s=1, \ldots, N$, let $w_{I}^{s} \in V_{h}^{s}$ be the piecewise linear interpolant of $w$, so that interpolation estimate (3.6) holds, and let $\widetilde{w}_{I} \in \Phi_{h}$ be the piecewise linear interpolant of $w$ on $\Sigma$, for which interpolation estimate (3.8) holds. Then, integrating by parts, inserting $w_{I}^{s}$, using (3.1] $\left.\mathrm{i}\right)$, and inserting $\widetilde{w}_{I}$, we get

$$
\begin{aligned}
\left\|u-u_{h}\right\|_{0, \Omega}^{2}= & \left\langle u-u_{h}, A^{*} w\right\rangle \\
= & -\sum_{s}\left\langle u-u_{h}, \frac{\partial w}{\partial n_{a}}\right\rangle_{s}+\sum_{s} a_{s}\left(u-u_{h}, w\right) \\
= & I+\sum_{s} a_{s}\left(u-u_{h}, w-w_{I}^{s}\right)+\sum_{s} a_{s}\left(u-u_{h}, w_{I}^{s}\right) \\
= & I+I I+\sum_{s}\left\langle\lambda-\lambda_{h}, w_{I}^{s}\right\rangle_{s} \\
= & I+I I+\sum_{s}\left\langle\lambda-\lambda_{h}, w_{I}^{s}-w\right\rangle_{s} \\
& +\sum_{s}\left\langle\lambda-\lambda_{h}, w-\widetilde{w}_{I}\right\rangle_{s}+\sum_{s}\left\langle\lambda-\lambda_{h}, \widetilde{w}_{I}\right\rangle_{s} \\
= & I+I I+I I I+I V+V .
\end{aligned}
$$

The term $I$ will be estimated later on. To estimate $I I$ we proceed as for (3.28) and use continuous dependence of $w$ on $u-u_{h}$ and (3.44). Thus,

$$
I I \leq C \bar{h}|w|_{2, \Omega}\left(\sum_{s}\left\|u-u_{h}\right\|_{1, \Omega^{s}}^{2}\right)^{1 / 2} \leq C \bar{h} E_{h}\left\|u-u_{h}\right\|_{0, \Omega} .
$$

The term $V$ vanishes thanks to (3.1 iii), while $I I I$ and $I V$ can be treated exactly as (3.29) and (3.32) respectively:

$$
\begin{aligned}
I I I, I V & \leq C \sum_{s} \sum_{T}\left(h_{T}^{2}|u|_{2, T}+h_{T}|| u-u_{h} \|_{1, T}\right)|w|_{2, T} \\
& \leq C \sum_{s} \sum_{T}\left(h_{T}|u|_{2, T}+\left\|u-u_{h}\right\|_{1, T}\right) \bar{h}|w|_{2, T} \\
& \leq C\left(E_{I}^{2}+E_{h}^{2}\right)^{1 / 2} \bar{h}|w|_{2, \Omega} \leq C \bar{h} E_{I}|| u-u_{h} \|_{0, \Omega} .
\end{aligned}
$$

It remains to estimate the term $I$. For that, set $\mu_{s}^{*}=\partial w / \partial n_{a \mid \Gamma^{s}}$ for every $s=1, \ldots, N$. Let $\mu_{I}^{s}$ be the interpolant of $\mu_{s}^{*}$ defined as in (3.9). Inserting $\mu_{I}^{s}$ and using (3.1) ii), (3.13), (3.11), and (2.8), we have

$$
\begin{aligned}
I & =-\sum_{s}\left\langle u-u_{h}, \mu_{s}^{*}-\mu_{I}^{s}\right\rangle_{s}-\sum_{s}\left\langle u-u_{h}, \mu_{I}^{s}\right\rangle_{s} \\
& =-\sum_{s}\left\langle u-u_{h}, \mu_{s}^{*}-\mu_{I}^{s}\right\rangle_{s}-\sum_{s}\left\langle\psi-\psi_{h}, \mu_{I}^{s}\right\rangle_{s} \\
& =-\sum_{s}\left\langle u-u_{h}, \mu_{s}^{*}-\mu_{I}^{s}\right\rangle_{s} \leq \sum_{s}\left|u-u_{h}\right|_{1 / 2, \Gamma^{s}}|| \mu_{s}^{*}-\left.\mu_{I}^{s}\right|_{-1 / 2, \Gamma^{s}} \\
& \leq C \sum_{s}\left|u-u_{h}\right|_{1, \Omega^{s}} \bar{h}\left|\mu_{s}^{*}\right|_{1 / 2, \Gamma^{s}} \leq C \bar{h} E_{h}|w|_{2, \Omega} \leq C \bar{h} E_{I}|| u-u_{h} \|_{0, \Omega} .
\end{aligned}
$$


Finally, using (3.47)-(3.49) in (3.46), we reach the desired conclusion.

So far, we have obtained optimal error estimates for the variable $u$. In a sense, this might be considered as sufficient to assess the accuracy of the three-field formulation. However, it might be interesting to see if similar error estimates could be proved for the other two variables $\lambda$ and $\psi$. In the sequel we are going to present some of those error estimates, restraining ourselves, for the sake of simplicity, to the ones whose proof is more elementary. In particular we shall obtain optimal error estimates for both $\psi$ and $\lambda$ in the weighted $L^{2}$-norms:

$$
\|\left.|\varphi|\right|_{\Sigma_{\psi}}=\left(\sum_{I_{\psi}}\left|I_{\psi}\right|\|\varphi\|_{0, I_{\psi}}^{2}\right)^{1 / 2}
$$

and

$$
|\| \mu|||_{\Sigma_{\lambda}}=\left(\sum_{s} \sum_{k}\left|I_{k}\right|\|\mu\|_{0, I_{k}}^{2}\right)^{1 / 2}
$$

In order to make the following exposition more fluent, we anticipate the following technical fact, whose proof is totally elementary and will therefore be left to the reader. Assume that $I$ is an interval, $\rho$ a real number with $0<\rho<1$, and $I_{\rho}$ a subinterval having length $\left|I_{\rho}\right| \geq \rho|I|$. Let $m_{\rho}$ be the midpoint of $I_{\rho}$. Then, for every polynomial $p$ of degree $\leq 1$ that does not change sign in $I$, we have

$$
\int_{I_{\rho}} p\left(m_{\rho}\right) p(t) d t=\left|I_{\rho}\right| p^{2}\left(m_{\rho}\right) \geq \frac{3 \rho^{3}}{4} \int_{I} p^{2}(t) d t .
$$

We can now prove the following inf-sup condition for the spaces $M_{h}^{s}$ and $\Phi_{h \mid \Gamma^{s}}$.

Lemma 5. Let $s$ be fixed. Assume that the interval $I_{\psi} \in \mathcal{T}_{\psi_{\mid \Gamma s}}$ contains at least two regular intervals $I_{\lambda} \in \mathcal{T}_{\lambda}^{s}$. Then, for every $\varphi_{h} \in \Phi_{h}$ not identically zero on $I_{\psi}$, there exists a $\mu_{h}^{s} \in M_{h}^{s}$, not identically zero on $I_{\psi}$, such that

$$
\left\|\mu_{h}^{s}\right\|_{0, I_{\psi}}^{2}=\int_{I_{\psi}} \mu_{h}^{s} \varphi_{h} d \Gamma \geq\left(\frac{3 \rho_{\min }^{3}}{4}\right)^{1 / 2}\left\|\varphi_{h}\right\|_{0, I_{\psi}}\left\|\mu_{h}^{s}\right\|_{0, I_{\psi}},
$$

where

$$
\rho_{\min }=\min \left\{\frac{\beta h}{\left|I_{\psi}\right|}, \quad I_{\psi} \subset \mathcal{T}_{\psi \mid \Gamma^{s}}\right\},
$$

and $\beta$ is defined in (2.18).

Proof. As $\varphi_{h}$ is not identically zero, there exists at least one regular interval $I_{\lambda}$ where $\varphi_{h}$ does not change sign. Let $m$ be the midpoint of this $I_{\lambda}$, and set $\mu_{h}^{s}=$ $\varphi_{h}(m)$ in $I_{\lambda}, \mu_{h}^{s}=0$ in the rest of $I_{\psi}$. We then have

$$
\int_{I_{\psi}} \mu_{h}^{s} \varphi_{h} d \Gamma=\left\|\mu_{h}^{s}\right\|_{0, I_{\psi}}^{2}
$$

On the other hand, using (3.52), we also have

$$
\int_{I_{\psi}} \mu_{h}^{s} \varphi_{h} d \Gamma \geq \frac{3 \rho_{\min }^{3}}{4}\left\|\varphi_{h}\right\|_{0, I_{\psi}}^{2}
$$

as the regularity of $I_{\lambda}$ implies $\left|I_{\lambda}\right| \geq \beta h \geq \rho_{\min }\left|I_{\psi}\right|$. Now (3.55)-(3.56) give (3.53).

We can now prove our convergence theorem for the variable $\psi$. 
Theorem 3. Let $(u, \lambda, \psi)$ and $\left(u_{h}, \lambda_{h}, \psi_{h}\right)$ be the solutions of (1.2) and (2.23) respectively, and assume that for all $s=1, \ldots, N$ each interval $I_{\psi} \in \mathcal{T}_{\psi_{\mid \Gamma^{s}}}$ contains at least two regular intervals $I_{\lambda} \in \mathcal{T}_{\lambda}^{s}$. Then

$$
\|\left|\psi-\psi_{h}\right|||_{\Sigma_{\psi}}=\left(\sum_{s} \sum_{I_{\psi}}\left|I_{\psi}\right||| \psi-\left.\psi_{h}\right|_{0, I_{\psi}} ^{2}\right)^{1 / 2} \leq C \bar{h} E_{I} \leq C \bar{h}^{2}|u|_{2, \Omega} .
$$

Proof. Let $\psi_{I}$ be the interpolant of $\psi$ as in (3.7), and use, in every $I_{\psi}$, Lemma 5 with $\varphi_{h}=\psi_{h}-\psi_{I}$. We obtain

$$
\left|I_{\psi}\right|^{1 / 2} \int_{I_{\psi}} \mu_{h}^{s}\left(\psi_{h}-\psi_{I}\right) d \Gamma \geq\left(\frac{3 \rho_{\min }^{3}}{4}\right)^{1 / 2}\left|I_{\psi}\right|^{1 / 2}|| \psi_{h}-\psi_{I}\left\|_{0, I_{\psi}}\right\| \mu_{h}^{s} \|_{0, I_{\psi}} .
$$

On the other hand,

$$
\begin{aligned}
& \left|I_{\psi}\right|^{1 / 2} \int_{I_{\psi}} \mu_{h}^{s}\left(\psi_{h}-\psi_{I}\right) d \Gamma \\
& \quad=\left|I_{\psi}\right|^{1 / 2} \int_{I_{\psi}} \mu_{h}^{s}\left(\psi_{h}-\psi\right) d \Gamma+\left|I_{\psi}\right|^{1 / 2} \int_{I_{\psi}} \mu_{h}^{s}\left(\psi-\psi_{I}\right) d \Gamma=I+I I .
\end{aligned}
$$

In order to bound the first term, as in (3.30) we take $T_{\psi}$ a triangle in $\Omega^{s}$ having $I_{\psi}$ as an edge, and we apply (2.11) in $T_{\psi}$. We obtain

$$
\begin{aligned}
I & =\left|I_{\psi}\right|^{1 / 2} \int_{I_{\psi}} \mu_{h}^{s}\left(\psi_{h}-\psi\right) d \Gamma=\left|I_{\psi}\right|^{1 / 2} \int_{I_{\psi}} \mu_{h}^{s}\left(u_{h}-u\right) d \Gamma \\
& \leq\left|I_{\psi}\right|^{1 / 2}\left\|\mu_{h}^{s}\right\|_{0, I_{\psi}}\left\|u_{h}-u\right\|_{0, I_{\psi}} \\
& \leq C\left|I_{\psi}\right|^{1 / 2}\left\|\mu_{h}^{s}\right\|_{0, I_{\psi}}\left(\left|I_{\psi}\right|^{-1}|| u-u_{h}\left\|_{0, T_{\psi}}^{2}+\right\| u-u_{h}\left\|_{0, T_{\psi}}|| u-u_{h}\right\|_{1, T_{\psi}}\right)^{1 / 2} .
\end{aligned}
$$

The second term in (3.59) is easily bounded using (3.8) and (2.10):

$$
I I \leq\left. C|| \mu_{h}^{s}\right|_{0, I_{\psi}}\left|I_{\psi}\right|^{2}|\psi|_{3 / 2, I_{\psi}} \leq C|| \mu_{h}^{s} \|_{0, I_{\psi}}\left|I_{\psi}\right|^{2}|u|_{2, T_{\psi}} .
$$

Inserting (3.60)-(3.61) in (3.59) and then in (3.58) and taking the square gives, on the intervals $I_{\psi}$ where $\psi_{h}-\psi_{I}$ does not vanish identically,

$$
\begin{aligned}
& \left|I_{\psi}\right|\left\|\psi_{h}-\psi_{I}\right\|_{0, I_{\psi}}^{2} \\
& \quad \leq C\left(\left\|u-u_{h}\right\|_{0, T_{\psi}}^{2}+\left|I_{\psi}\right||| u-u_{h}\left\|_{0, T_{\psi}}|| u-u_{h}\right\|_{1, T_{\psi}}+\left|I_{\psi}\right|^{4}|u|_{2, T_{\psi}}^{2}\right) .
\end{aligned}
$$

Summation of (3.62) gives, using (3.44) and (3.45),

$$
\sum_{s} \sum_{I_{\psi}}\left|I_{\psi}\right|\left\|\psi_{h}-\psi_{I}\right\|_{0, I_{\psi}}^{2} \leq C \bar{h}^{2} E_{I}^{2}
$$

and the result follows by (3.8) and the triangle inequality.

Remark 3. We notice that the additional assumption required on $\mathcal{T}_{\lambda}^{s}$ for proving the error bound (3.57) is not difficult to realize in practice. Indeed, one can start by defining $\mathcal{T}_{\lambda}^{s}$ as in (2.17). If there is an $I_{\psi}$ that does not contain two regular intervals of $\mathcal{T}_{\lambda}^{s}$, we just add a node to $\mathcal{T}_{\lambda}^{s}$, by splitting into two equal parts the longest $I_{\lambda}$ contained in $I_{\psi}$. 


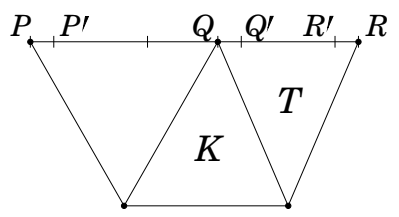

FIGURE 2.

Remark 4. The norm (3.50) used in estimate (3.57) is clearly weaker than the norm in $L^{2}(\Sigma)$. However it is optimal, compared with the regularity assumptions on the solution. In particular, using (2.16) and (3.57), one can easily deduce that

$$
\left\|\psi-\psi_{h}\right\|_{0, \Sigma} \leq C \bar{h}^{3 / 2}|u|_{2, \Omega}
$$

for quasi-uniform meshes.

As far as the $\lambda$-variable is concerned, we already had in (3.15) that

$$
\left|\lambda_{h}-\widetilde{\lambda}\right|_{\mid I_{k}} \leq C h_{T}{ }^{1 / 2}\left|I_{k}\right|^{-3 / 2}|| u-\left.u_{h}\right|_{1, T}
$$

for every $I_{k} \subset \mathcal{T}_{\lambda}^{s}(s=1, \ldots, N)$, where $T$ is the triangle in $\mathcal{T}_{u}^{s}$ having $I_{k}$ as part of its boundary. If $I_{k}$ is regular, this implies

$$
\left|\lambda_{h}-\widetilde{\lambda}\right|_{\mid I_{k}} \leq C\left|I_{k}\right|^{-1}|| u-u_{h} \|_{1, T} .
$$

We would like to prove that (3.66) is essentially true for irregular intervals as well. Assume therefore that $I_{k}$ is irregular. We need some notation. Let $Q$ be the endpoint of $I_{k}$ that is a vertex of $\mathcal{T}_{u}^{s}$ (there must be one). Let $T$ be the triangle of $\mathcal{T}_{u}^{s}$ having $I_{k} \subset \partial T$. Let $P$ and $R$ be the boundary vertices in $\mathcal{T}_{u}^{s}$ sharing a triangle with $Q$. Let $Q^{\prime}$ be the other endpoint of $I_{k}$ and assume, to fix ideas, that $Q^{\prime} \in \overline{Q R}$. As it will be the worst case, we also assume that there exist two other points, $P^{\prime} \in \overline{P Q}$ and $R^{\prime} \in \overline{Q R}$, such that $\overline{P P^{\prime}}$ and $\overline{R R^{\prime}}$ are irregular intervals of $\mathcal{T}_{\lambda}^{s}$. See Figure 2, Now let $v_{h}$ be a function in $V_{h}^{s}$ (hence, piecewise linear on $\mathcal{T}_{u}^{s}$ ) such that $v_{h}(Q)=1$ and $v_{h}=0$ at the other vertices, and $K=\operatorname{supp}\left(v_{h}\right)$. In the case of Figure 2, $K$ will be the union of the three triangles.

We are ready. Splitting the integral into five pieces, we have

$$
\begin{aligned}
\left|\int_{Q}^{Q^{\prime}}\left(\lambda_{h}-\widetilde{\lambda}\right) v_{h} d \Gamma\right| & =\left|\int_{P}^{R} \cdot d \Gamma-\int_{P}^{P^{\prime}} \cdot d \Gamma-\int_{P^{\prime}}^{Q} \cdot d \Gamma-\int_{Q^{\prime}}^{R^{\prime}} \cdot d \Gamma-\int_{R^{\prime}}^{R} \cdot d \Gamma\right| \\
& \leq|I|+|I I|+|I I I|+|I V|++|V|
\end{aligned}
$$

and we bound each of the five pieces separately. To start with, we remark that $I I I$ and $I V$ are made of regular intervals. Therefore from (3.66) we immediately have

$$
|I I I|+|I V| \leq C|| u-u_{h} \|_{1, K},
$$

as $\left|v_{h}\right| \leq 1$. On the other hand, using elementary calculus and then (3.65), we have

$|I I|=\left|\lambda_{h}-\widetilde{\lambda}\right|_{P P^{\prime}} \cdot\left|P P^{\prime}\right| \cdot v_{h}\left(\frac{P+P^{\prime}}{2}\right)=\left|\lambda_{h}-\widetilde{\lambda}\right|_{P P^{\prime}} \cdot \frac{\left(\left|P P^{\prime}\right|\right)^{2}}{2|P Q|} \leq C\left\|u-u_{h}\right\|_{1, K}$.

The term $V$ is then estimated in an identical way:

$$
|V| \leq C\left\|u-u_{h}\right\|_{1, K}
$$


so that we only have to deal with $I$. Adding and subtracting $\lambda$, using (3.12), (3.17), (2.9), and estimating the norms of $v_{h}$ (that is, $\left\|v_{h}\right\|_{0, P R} \leq C h^{1 / 2}$ and $\left\|v_{h}\right\|_{1, K} \leq C$ ), we obtain

$$
\begin{aligned}
\int_{P}^{R}\left(\lambda_{h}-\tilde{\lambda}\right) v_{h} d \Gamma & =\int_{P}^{R}\left(\lambda_{h}-\lambda\right) v_{h} d \Gamma+\int_{P}^{R}(\lambda-\tilde{\lambda}) v_{h} d \Gamma \\
& \leq C h^{1 / 2}|\lambda|_{1 / 2, P R}\left\|v_{h}\right\|_{0, P R}+a\left(u-u_{h}, v_{h}\right) \\
& \leq C h|u|_{2, K}+M\left\|u-u_{h}\right\|_{1, K}\left\|v_{h}\right\|_{1, K} \\
& \leq C\left(h|u|_{2, K}+\left\|u-u_{h}\right\|_{1, K}\right) .
\end{aligned}
$$

Summing all the five contributions from (3.68), (3.70), (3.69) and (3.71), we have

$$
\left|\int_{Q}^{Q^{\prime}}\left(\lambda_{h}-\tilde{\lambda}\right) v_{h} d \Gamma\right| \leq C\left(h|u|_{2, K}+\left\|u-u_{h}\right\|_{1, K}\right) .
$$

On the other hand, the same integral can be estimated from below:

$$
\begin{aligned}
\left|\int_{Q}^{Q^{\prime}}\left(\lambda_{h}-\widetilde{\lambda}\right) v_{h} d \Gamma\right| & =\left|\lambda_{h}-\widetilde{\lambda}\right|_{I_{k}} \cdot\left|Q Q^{\prime}\right| \cdot v_{h}\left(\frac{\left.Q+Q^{\prime}\right)}{2}\right) \\
& =\left|\lambda_{h}-\widetilde{\lambda}\right|_{I_{k}} \cdot\left|Q Q^{\prime}\right| \cdot \frac{2|Q R|-\left|Q Q^{\prime}\right|}{2|Q R|} \\
& \geq C\left|I_{k}\right|\left|\lambda_{h}-\widetilde{\lambda}\right|_{I_{k}} .
\end{aligned}
$$

Comparing (3.72) and (3.73), we conclude with the following theorem.

Theorem 4. Let $(u, \lambda, \psi)$ and $\left(u_{h}, \lambda_{h}, \psi_{h}\right)$ be the solutions of (1.2) and (2.23) respectively, and let $\tilde{\lambda}$ be defined by (3.10). For all $s=1, \ldots, N$ let $I_{k}$ be an interval in $\mathcal{T}_{\lambda}^{s}$, and let $K$ be the union of those triangles of $\mathcal{T}_{u}^{s}$ that have at least one point in common with $I_{k}$. We have

$$
\left|\lambda_{h}-\widetilde{\lambda}\right|_{I_{k}} \leq C\left|I_{k}\right|^{-1}\left(h|u|_{2, K}+|| u-\left.u_{h}\right|_{1, K}\right) .
$$

From (3.74), the previous error estimate (3.44), the interpolation estimate (3.12) and the triangle inequality we have then the final error estimate for $\lambda$, in the norm (3.51).

Theorem 5. Let $(u, \lambda, \psi)$ and $\left(u_{h}, \lambda_{h}, \psi_{h}\right)$ be the solutions of (1.2) and (2.23) respectively. We have

$$
\|\left|\lambda-\lambda_{h}\right|||_{\Sigma_{\lambda}}=\left(\sum_{s} \sum_{k}\left|I_{k}\right|\left\|\lambda-\lambda_{h}\right\|_{0, I_{k}}^{2}\right)^{1 / 2} \leq C E_{I} \leq C \bar{h}|u|_{2, \Omega} .
$$

\section{NUMERICAL CONSIDERATIONS}

In this section we shall address a few numerical considerations, in order to cast some light on different issues, like the actual implementation of the procedure and its expected numerical performance. To present a full set of numerical experiments (that might show the accuracy and stability of the method, the quality of the preconditioners that can be used, and the overall performance in terms of accuracy versus computer time in different typical situations of industrial interest) is far beyond the scope of this paper.

On the other hand, since full numerical evidence in support of the present approach is presently lacking, it is worth a few comments to explain why we believe in its potential. 
We then go back to the stabilized problem (2.23), which we report here for convenience:

$$
\begin{cases}\text { find } u_{h} \in \widetilde{V}_{h}^{*}, \lambda_{h} \in M_{h}^{*} \text { and } \psi_{h} \in \Phi_{h} \text { such that } \\ \text { i) } a_{s}\left(u_{h}^{s}, v\right)-\left\langle\lambda_{h}^{s}, v\right\rangle_{s}=(f, v)_{s}, & \forall v \in \widetilde{V}_{h}^{s}, \forall s, \\ \text { ii) }\left\langle\mu^{s}, u_{h}^{s}\right\rangle_{s}=\left\langle\mu^{s}, \psi_{h}\right\rangle_{s}, & \forall \mu^{s} \in M_{h}^{s}, \forall s, \\ \text { iii) } \sum_{s}\left\langle\lambda_{h}^{s}, \varphi\right\rangle_{s}=0, & \forall \varphi \in \Phi_{h} .\end{cases}
$$

First of all, we note that the formulation (4.1) does not seem to be very well suited for a global solution, as one would have by applying an iterative algorithm (conjugate gradient, GMRES, etc.) to the whole system. Even the procedure that eliminates the bubbles and the Lagrange multipliers $\lambda_{h}^{s}$ (which we are going to describe in detail in the sequel) will not produce a system which is well suited for that. On the other hand, in most cases, this is not the preferred way for solving a domain decomposition problem. Looking at (4.1), it is clear that, for fixed $\psi_{h}$, the first two equations can be solved independently and in parallel. We can therefore consider the mapping $\mathcal{S}_{h}=\left(\mathcal{S}_{u}, \mathcal{S}_{\lambda}\right)$ that associates to the pair $\left(f, \psi_{h}\right)$ the solution $\left(u_{h}, \lambda_{h}\right)$ of the first two equations of (4.1). With this notation, problem (4.1) can be written as

$$
\text { find } \psi_{h} \in \Phi_{h} \text { such that } \quad \sum_{s}\left\langle\mathcal{S}_{\lambda}\left(f, \psi_{h}\right), \varphi\right\rangle_{s}=0, \quad \forall \varphi \in \Phi_{h} .
$$

It is clear that in (4.2) a crucial role is played by the linear operator $S_{h}$, from $\Phi_{h}$ to its dual space, defined by:

$$
\left\langle S_{h}\left(\psi_{h}\right), \varphi\right\rangle:=\sum_{s}\left\langle\mathcal{S}_{\lambda}\left(0, \psi_{h}\right), \varphi\right\rangle_{s}
$$

which is commonly called the Schur complement, and whose spectral properties have a paramount relevance in solving (4.2) by iterative methods. It is easy to see that $S_{h}$ is the discretization of a pseudo-differential operator $S$, of order 1 , on $\Sigma_{h}$. In order to precondition (4.2) one has to find a cheaply computable operator that could be regarded as an approximation of $S^{-1}$. Several choices for that can be found in the literature. For instance one could solve local Neumann problems (not necessarily related to the actual form of the operator $A$ or to the $\mathcal{T}_{u}^{s}$ grids) in order to get an approximation of the local Steklov-Poincaré operators. Or one can define a sort of $H^{1 / 2}$ inner product on $\Phi_{h}$, and invert the associated linear operator. We refer to the survey [11, and to the impressive set of proceedings of the various meetings on Domain Decomposition Methods, whose latest volume is 4]. It is clear, however, that in doing that the task is made much easier if the grid on $\Sigma_{h}$ is uniform. For instance, one could use fast solvers for the local Neumann problems, or find easy expressions for the $H^{1 / 2}$ inner product, and so on. This is the main reason why we believe that an approach allowing the use of a uniform grid on $\Sigma_{h}$ (independently of the grids that are used in the subdomains) is worth investigating.

Let us now discuss the actual computation of the operator $\mathcal{S}_{h}$, and in particular the solution of the local problems in each $\Omega^{s}$. For this, we concentrate on a single domain. We assume $\psi_{h}$ to be given, and we see how to compute the corresponding $u_{h}^{s}$ and $\lambda_{h}^{s}$. For simplicity, we drop the superscript $s$, as the same identical procedure will be applied, in parallel, in each subdomain. With an abuse of notation, we are also going to call the current subdomain $\Omega$ (instead of $\Omega^{s}$ ), and so on. No confusion 
should arise. With this simplified notation the local problem becomes

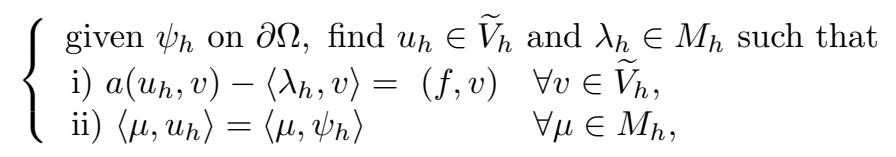

where $M_{h}$ is made of piecewise constants on $\mathcal{T}_{\lambda}$, and $\widetilde{V}_{h}$ is obtained as the sum of piecewise linear functions on $\mathcal{T}_{u}$ (which we will denote by $V_{L}$ ), and the space of boundary bubbles $B_{h}$. Accordingly, $u_{h}$ will be written as

$$
u_{h}(x)=u_{L}(x)+\sum_{k} u_{B}^{k} b_{k}(x)
$$

From the second equation of (4.4), by taking $\mu$ as the characteristic function of an interval $I_{k}$ of $\mathcal{T}_{\lambda}$, we obtain

$$
u_{B}^{k}=\left(\int_{I_{k}} \psi_{h} d s-\int_{I_{k}} u_{L} d s\right) / \int_{I_{k}} b_{k} d s
$$

We remark that both $u_{L}$ and $\psi_{h}$ are linear in each $I_{k}$, so that, indicating by $m_{k}$ the midpoint of $I_{k}$, and setting

$$
\gamma_{k}^{I}=\int_{I_{k}} b_{k} d s
$$

we immediately obtain from (4.6) that

$$
u_{B}^{k}=\left(\psi_{h}\left(m_{k}\right)-u_{L}\left(m_{k}\right)\right)\left|I_{k}\right| / \gamma_{k}^{I} .
$$

We can now use the first equation of (4.4), with $v=b_{k}$, in order to express $\lambda_{k}$ as a function of the other variables:

$$
\lambda_{k}=\left(a\left(u_{L}, b_{k}\right)+u_{B}^{k} a\left(b_{k}, b_{k}\right)-\left(f, b_{k}\right)\right) / \gamma_{k}^{I} .
$$

Assume now, for simplicity, that in (2.2) we have $a_{0}=0$, and that the other coefficients $a_{i, j}$ are piecewise constant. Assume also that the right-hand side $f$ is piecewise constant. The last two assumptions are not really restrictive, as in most cases both $a_{i, j}$ and $f$ are approximated anyhow by piecewise constants in the actual implementation. The first assumption, on $a_{0}$, is more restrictive, but, as we shall see, is there only to have a nicer final formula, and a piecewise constant $a_{0}$ could also be taken very easily into account. We remark now that, integrating by parts, for every $v_{L} \in V_{L}$ and for every $k$ we have

$$
a\left(v_{L}, b_{k}\right)=a\left(b_{k}, v_{L}\right)=\int_{I_{k}} \frac{\partial v_{L}}{\partial n_{a}} b_{k} d s=\frac{\partial v_{L}}{\partial n_{a}} \gamma_{k}^{I},
$$

where clearly $\partial v_{L} / \partial n_{a}$ is the conormal derivative of $v_{L}$ with respect to the bilinear form $a$, as given in (2.3). We also set

$$
\gamma_{k}^{T}=\int_{T_{k}} b_{k} d x, \quad \gamma_{k}^{a}=a\left(b_{k}, b_{k}\right),
$$

so that (4.9) becomes

$$
\lambda_{k}=\frac{\partial u_{L}}{\partial n_{a}}+\frac{\left(u_{B}^{k} \gamma_{k}^{a}-f_{k} \gamma_{k}^{T}\right)}{\gamma_{k}^{I}} .
$$


With some manipulations, using (4.8) and (4.10), we obtain

$$
a\left(\sum_{k} u_{B}^{k} b_{k}, v_{L}\right)=\int_{\partial \Omega}\left(\psi_{h}-u_{L}\right) \frac{\partial v_{L}}{\partial n_{a}} d s .
$$

Taking now the first equation of (4.4) for $v=v_{L}$, using (4.8), (4.12) and (4.10), we obtain with easy computations that

$$
\begin{aligned}
& a\left(u_{L}, v_{L}\right)-\left\langle u_{L}, \frac{\partial v_{L}}{\partial n_{a}}\right\rangle-\left\langle v_{L}, \frac{\partial u_{L}}{\partial n_{a}}\right\rangle+\sum_{k}\left(\frac{\left|I_{k}\right|}{\gamma_{k}^{I}}\right)^{2} \gamma_{k}^{a} u_{L}\left(m_{k}\right) v_{L}\left(m_{k}\right) \\
& =\left(f, v_{L}\right)-\sum_{k} f_{k}\left(\frac{\gamma_{k}^{T}\left|I_{k}\right|}{\gamma_{k}^{I}}\right) v_{L}\left(m_{k}\right)-\left\langle\psi_{h}, \frac{\partial v_{L}}{\partial n_{a}}\right\rangle \\
& \quad+\sum_{k}\left(\frac{\left|I_{k}\right|}{\gamma_{k}^{I}}\right)^{2} \gamma_{k}^{a} \psi_{h}\left(m_{k}\right) v_{L}\left(m_{k}\right) .
\end{aligned}
$$

Equation (4.14) can be further simplified. In particular, if $v_{L}$ is a basis function having value 1 at a boundary node and 0 at the other nodes of $\mathcal{T}_{u}$, and if $f$ is piecewise constant on $\mathcal{T}_{u}$, using (2.20) we rather easily get

$$
\sum_{k} f_{k}\left(\frac{\gamma_{k}^{T}\left|I_{k}\right|}{\gamma_{k}^{I}}\right) v_{L}\left(m_{k}\right)=\sum_{k} f_{k} \frac{\left|T_{k}\right|}{2} v_{L}\left(m_{k}\right)=\frac{3}{4} \int f v_{L} d x,
$$

where the last integral is over the triangles having an edge on $\partial \Omega$. By linearity, the formula will then hold for every $v_{L}$ vanishing at all internal nodes. Hence, we can also consider the new scalar product, defined for piecewise linear functions $u_{L}$ and $v_{L}$ as

$$
\left\langle u_{L}, v_{L}\right\rangle_{h}:=\sum_{k}\left(\frac{\left|I_{k}\right|}{\gamma_{k}^{I}}\right)^{2} \gamma_{k}^{a} u_{L}\left(m_{k}\right) v_{L}\left(m_{k}\right),
$$

and, in agreement with (4.15),

$$
\left(f, v_{L}\right)_{*}:=\frac{3}{4} \int f v_{L}^{*} d x,
$$

where the integral is extended over the elements having an edge on $\partial \Omega$, and $v_{L}^{*}$ is obtained from $v_{L}$ by setting all the values at internal nodes equal to zero. Equation (4.14) now becomes

$$
\begin{aligned}
& a\left(u_{L}, v_{L}\right)-\left\langle u_{L}, \frac{\partial v_{L}}{\partial n_{a}}\right\rangle-\left\langle v_{L}, \frac{\partial u_{L}}{\partial n_{a}}\right\rangle+\left\langle u_{L}, v_{L}\right\rangle_{h} \\
& \quad=\left(f, v_{L}\right)-\left(f, v_{L}\right)_{*}-\left\langle\psi_{h}, \frac{\partial v_{L}}{\partial n_{a}}\right\rangle+\left\langle\psi_{h}, v_{L}\right\rangle_{h} \quad \forall v_{L} \in V_{L} .
\end{aligned}
$$

The nature of the resulting local problem should now be clear. We remark first that the method we obtained by adding and eliminating bubbles is very close to Nitsche's method [17, which roughly corresponds to

$$
\begin{aligned}
& a\left(u_{L}, v_{L}\right)-\left\langle u_{L}, \frac{\partial v_{L}}{\partial n_{a}}\right\rangle-\left\langle v_{L}, \frac{\partial u_{L}}{\partial n_{a}}\right\rangle+\frac{\alpha}{h}\left\langle u_{L}, v_{L}\right\rangle \\
& \quad=\left(f, v_{L}\right)-\left\langle\psi_{h}, \frac{\partial v_{L}}{\partial n_{a}}\right\rangle+\frac{\alpha}{h}\left\langle\psi_{h}, v_{L}\right\rangle \quad \forall v_{L} \in V_{L},
\end{aligned}
$$

and whose implementation is immediate. In (4.19) $\alpha$ is a suitable constant which has to be $\geq \alpha_{0}$, depending on the minimum angle of the triangles. We also point out that, for reasonably smooth $f$, the term $\left(f, v_{L}\right)_{*}$, which appears in (4.18) and 


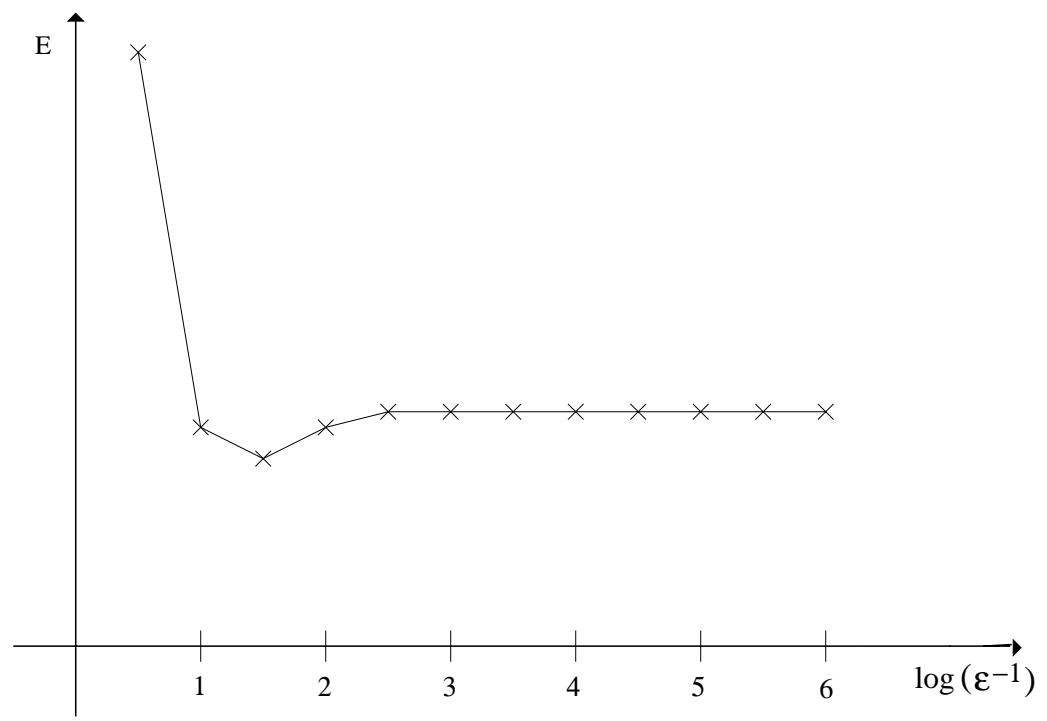

FiguRE 3.

not in (4.19), will actually be negligible (but in any case is easy to compute). On the other hand the terms $\frac{\alpha}{h}\left\langle u_{L}, v_{L}\right\rangle$ and $\left\langle u_{L}, v_{L}\right\rangle_{h}$ compare very well in terms of powers of $h$. The effect of having smaller intervals $I_{k}$, or even irregular intervals, should correspond, roughly speaking, to choosing a local value for $\alpha$ which is bigger than necessary. In these circumstances, both (4.18) and (4.19) exhibit, in practical computations, the typical behavior of penalty methods, as depicted in Figure 3: for smaller and smaller values of the penalty parameter $\varepsilon$, the relative error stabilizes to a value which is slightly bigger that the optimal one (corresponding, in general, to $\varepsilon \sim h$ ) that is well within the bounds of optimality (in terms of powers of $h$ ). For this reason, we believe that our assumptions on the grids, and in particular the quasi-uniformity of $\mathcal{T}_{u}$ on the boundary and the comparability of the two grids $\mathcal{T}_{u}$ and $\mathcal{T}_{\psi}$, are rather technical assumptions, and do not correspond to an actual weakness of the method.

Finally, we want to point out that, in the spirit of [1], one could think of changing the shape of the bubbles $b_{k}$ so that the actual computation of the coefficients appearing in the final reduced system will become easier. Essentially, within certain limitations, one might prescribe the value of the coefficients and use them in the computation, just knowing that there exist bubbles (still providing optimal error bounds) that will produce, after elimination, the prescribed coefficients. The actual shape of these virtual bubbles does not need to be known explicitly, as only the final coefficients enter the computation. These matters are currently under investigation.

\section{REFERENCES}

1. C. Baiocchi, F. Brezzi, and L.P. Franca, Virtual bubbles and Ga.L.S., Comp. Meth. in Appl. Mech. and Eng., vol. 105, 1993, pp. 125-141. MR 94g:65058

2. C. Baiocchi, F. Brezzi, and L.D. Marini, Stabilization of Galerkin methods and applications to domain decomposition, in Future Tendencies in Computer Science, Control and Applied Mathematics, A. Bensoussan et al., ed., vol. 653, Lecture Notes in Computer Science, SpringerVerlag, 1992, pp. 345-355. MR 94g:65119 
3. P.E. Bjørstad and O.B. Widlund, Iterative methods for the solution of elliptic problems on regions partitioned into substructures, SIAM J. Numer. Anal., vol. 23, 1986, pp. 1093-1120. MR 88h:65188

4. P.E. Bjørstad, M.S. Espedal and D.E. Keyes (eds.), Domain Decomposition Methods in Science and Engineering, Domain Decomposition Press, Bergen, 1998.

5. J.H. Bramble, J. Pasciak and A. Schatz, The construction of preconditioners for elliptic problems by substructuring, IV, Math. Comput., vol. 53, 1989, pp. 1-24. MR 89m:65098

6. F. Brezzi and M. Fortin, Mixed and hybrid finite element methods, Springer-Verlag, New York, 1991. MR 92d:65187

7. F. Brezzi, L.P. Franca, L.D. Marini, and A. Russo, Stabilization techniques for domain decomposition methods with non-matching grids, in [4], pp. 1-11.

8. F. Brezzi and L. D. Marini, Macro hybrid elements and domain decomposition methods, in Optimization et Contrôle, J. Désidéri et al., ed., Toulouse, 1993, CÉPADUÈs-Editions, pp. 89-96. MR 95c:65208

9. — - A three-field domain decomposition method, in Domain Decomposition Methods in Science and Engineering, A. Quarteroni et al., ed., Contemp. Math., vol. 157, Amer. Math. Soc., Providence, RI, 1994, pp. 27-34. MR 95a:65202

10. A. Buffa, Error estimate for a stabilized domain decomposition method with nonmatching grid. Numer. Math., to appear.

11. T.F. Chan and T.P. Mathew, Domain decomposition algorithms, Acta Numerica 1994, pp. 61144. MR 95f:65214

12. P.G. Ciarlet, The finite element method for elliptic problems, North-Holland Publishing Company, Amsterdam, Holland, 1978. MR 58:25001

13. M. Dryja, A finite element-capacitance method for elliptic problems on regions partitioned into subregions, Numer. Math., vol. 44, 1984, pp. 153-168. MR 86c:65131

14. R. Glowinski, W.K. Kinton and M.F. Wheeler, Acceleration of domain decomposition algorithms for mixed finite elements by multilevel methods, in Third Int. Symp. on Domain Decomposition Methods for Partial Differential Equations, T. Chan et al., ed., SIAM (Philadelphia), 1990, pp. 263-289. MR 91e:65010

15. G. Golub and D. Mayers, The use of preconditioning over irregular regions, in Computing Methods in Applied Sciences and Engineering, VI, R. Glowinski and J.L. Lions, eds., North Holland, 1984, pp. 3-14. MR 87h:65059

16. Yu. A. Kuznetsov and M.F. Wheeler, Optimal order substructuring preconditioners for mixed finite element methods on nonmatching grids, East-West Journal of Numerical Mathematics, vol. 3, 1995, pp. 127-144. MR 96c:65182

17. J.A. Nitsche, Über ein Variationsprinzip zur Lösung Dirichlet-Problemen bei Verwendung von Teilräumen, die keinen Randbedingungen unterworfen sind, Abh. Math. Sem. Univ. Hamburg vol. 36, 1971, pp. 9-15. MR 49:6649

18. B.F. Smith and O.B. Widlund, A domain decomposition algorithm using a hierarchical basis, SIAM J. Sci. Comput., vol. 11, 1990, pp. 1212-1220. MR 91m:65125

19. C.H. Tong, T.F. Chan and C.C.J. Kuo, A domain decomposition preconditioner based on a change to a multilevel nodal basis, SIAM J. Sci. Comput., vol. 12, 1991, pp. 1486-1495. MR 92i: 65070

20. J. Xu, Theory of multilevel methods, PhD thesis, Cornell University, 1989.

Dipartimento di Matematica, Università di Pavia, via Ferrata 1, 27100 Pavia, Italy; Istituto di Analisi Numerica del CNR, via Ferrata 1, 27100 Pavia, Italy

E-mail address: brezzi@dragon.ian.pv.cnr.it

Dipartimento di Matematica, Università di Pavia, via Ferrata 1, 27100 Pavia, Italy; Istituto di Analisi Numerica del CNR, via Ferrata 1, 27100 Pavia, Italy

E-mail address: marini@dragon.ian.pv.cnr.it 